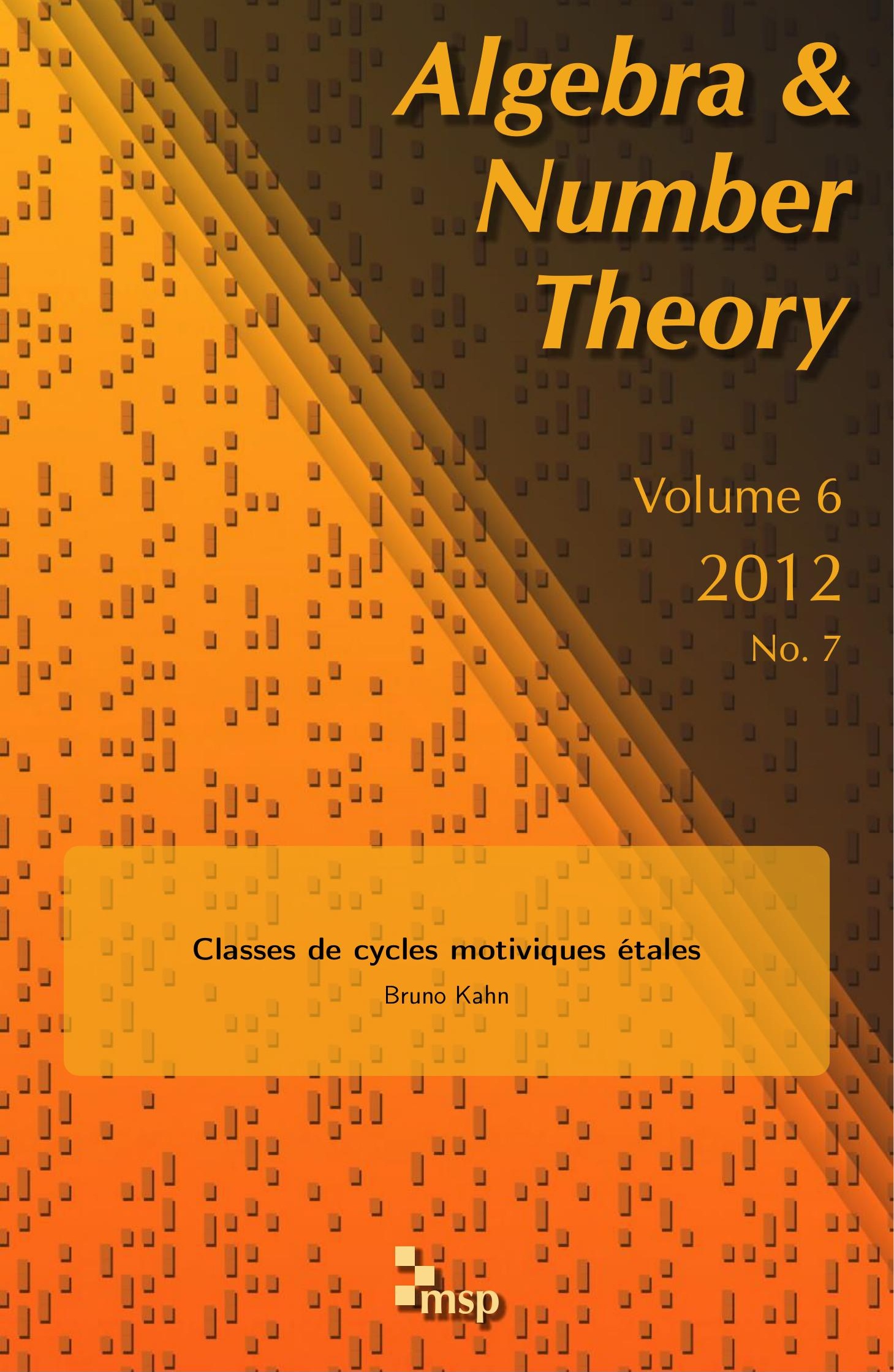




\title{
Classes de cycles motiviques étales
}

\author{
Bruno Kahn
}

Résumé. Soit $X$ une variété lisse sur un corps $k$ et soit $l$ un nombre premier. On construit une suite exacte

$$
0 \rightarrow H^{0}\left(X, \mathscr{H}_{\text {cont }}^{3}\left(\mathbb{Z}_{l}(2)\right)\right) \otimes \mathbb{Q} / \mathbb{Z} \rightarrow H^{0}\left(X, \mathscr{H}_{\mathrm{ett}}^{3}\left(\mathbb{Q}_{l} / \mathbb{Z}_{l}(2)\right)\right) \rightarrow C_{\text {tors }} \rightarrow 0
$$

où $\mathscr{H}_{\mathrm{ett}}^{i}\left(\mathbb{Q}_{l} / \mathbb{Z}_{l}(2)\right)$ et $\mathscr{H}_{\text {cont }}^{i}\left(\mathbb{Z}_{l}(2)\right)$ sont les faisceaux Zariski associés aux cohomologies étale et continue et $C$ est le conoyau de la classe de cycle $l$-adique définie par Jannsen sur $C H^{2}(X) \otimes \mathbb{Z}_{l}$ si $l \neq$ car $k$ et une variante de celle-ci si $l=$ car $k$. Si $k=\mathbb{C}$, cela fournit une autre démonstration d'un théorème de Colliot-Thélène-Voisin, qui évite l'utilisation de la conjecture de Bloch-Kato en degré 3 . Si $k$ est séparablement clos et $l \neq c a r k$, on obtient, toujours dans l'esprit de Colliot-Thélène et Voisin, une suite exacte

$$
\begin{aligned}
0 \rightarrow \operatorname{Griff}^{2}\left(X, \mathbb{Z}_{l}\right)_{\text {tors }} \rightarrow & H_{\text {tr }}^{3}\left(X, \mathbb{Z}_{l}(2)\right) \otimes \mathbb{Q} / \mathbb{Z} \\
& \rightarrow H^{0}\left(X, \mathscr{H}_{\text {cont }}^{3}\left(\mathbb{Z}_{l}(2)\right)\right) \otimes \mathbb{Q} / \mathbb{Z} \rightarrow \operatorname{Griff}^{2}\left(X, \mathbb{Z}_{l}\right) \otimes \mathbb{Q} / \mathbb{Z} \rightarrow 0
\end{aligned}
$$

où $H_{\mathrm{tr}}^{3}\left(X, \mathbb{Z}_{l}(2)\right)$ est le quotient de la cohomologie $l$-adique par le premier cran de la filtration par le coniveau et $\operatorname{Griff}^{2}\left(X, \mathbb{Z}_{l}\right)$ est un groupe de Griffiths $l$-adique.

Si $k$ est la clôture algébrique d'un corps fini et si $X$ est « de type abélien» et vérifie la conjecture de Tate, alors $\operatorname{Griff}^{2}\left(X, \mathbb{Z}_{l}\right)$ est de torsion et $H^{0}\left(X, \mathscr{H}_{\mathrm{ett}}^{3}\left(\mathbb{Q}_{l} / \mathbb{Z}_{l}(2)\right)\right)$ est fini si $H_{\mathrm{tr}}^{3}\left(X, \mathbb{Q}_{l}(2)\right)=0$. D'autre part, un théorème de Schoen donne un exemple où $H^{0}\left(X, \mathscr{H}_{\mathrm{et}}^{3}\left(\mathbb{Q}_{l} / \mathbb{Z}_{l}(2)\right)\right)$ est fini mais $H_{\mathrm{tr}}^{3}\left(X, \mathbb{Q}_{l}(2)\right) \neq 0$.

\section{An English abstract appears on the next page.}

1. Introduction

2. Groupes de Chow supérieurs

3. Cohomologie $l$-adique et $p$-adique

4. Cas d'un corps de base séparablement clos

5. Cas d'un corps de base fini et de sa clôture algébrique

Annexe: Cohomologie de Hodge-Witt logarithmique sur des corps imparfaits

Remerciements 1404

Bibliographie 1405

MSC2010 : primary 14C25; secondary 14E22, 14F20, 14F42, 14GXX.

Keywords : cycle class map, unramified cohomology, continuous étale cohomology, motivic cohomology. 
Abstract. Let $X$ be a smooth variety over a field $k$, and $l$ be a prime number. We construct an exact sequence

$$
0 \rightarrow H^{0}\left(X, \mathscr{H}_{\text {cont }}^{3}\left(\mathbb{Z}_{l}(2)\right)\right) \otimes \mathbb{Q} / \mathbb{Z} \rightarrow H^{0}\left(X, \mathscr{H}_{\mathrm{ett}}^{3}\left(\mathbb{Q}_{l} / \mathbb{Z}_{l}(2)\right)\right) \rightarrow C_{\text {tors }} \rightarrow 0
$$

where $\mathscr{H}_{\mathrm{ett}}^{i}\left(\mathbb{Q}_{l} / \mathbb{Z}_{l}(2)\right)$ and $\mathscr{H}_{\text {cont }}^{i}\left(\mathbb{Z}_{l}(2)\right)$ are the Zariski sheaves associated to étale and continuous étale cohomology and $C$ is the cokernel of Jannsen's $l$-adic cycle class on $C H^{2}(X) \otimes \mathbb{Z}_{l}$ if $l \neq$ char $k$ or a variant of it if $l=$ char $k$. If $k=\mathbb{C}$, this gives another proof of a theorem of Colliot-Thélène and Voisin, avoiding a recourse to the Bloch-Kato conjecture in degree 3 . If $k$ is separably closed and $l \neq$ char $k$, still in the spirit of Colliot-Thélène and Voisin we get an exact sequence

$$
\begin{aligned}
0 \rightarrow \operatorname{Griff}^{2}\left(X, \mathbb{Z}_{l}\right)_{\text {tors }} \rightarrow & H_{\text {tr }}^{3}\left(X, \mathbb{Z}_{l}(2)\right) \otimes \mathbb{Q} / \mathbb{Z} \\
& \rightarrow H^{0}\left(X, \mathscr{H}_{\text {cont }}^{3}\left(\mathbb{Z}_{l}(2)\right)\right) \otimes \mathbb{Q} / \mathbb{Z} \rightarrow \operatorname{Griff}^{2}\left(X, \mathbb{Z}_{l}\right) \otimes \mathbb{Q} / \mathbb{Z} \rightarrow 0
\end{aligned}
$$

where $H_{\mathrm{tr}}^{3}\left(X, \mathbb{Z}_{l}(2)\right)$ is the quotient of $l$-adic cohomology by the first step of the coniveau filtration and $\operatorname{Griff}^{2}\left(X, \mathbb{Z}_{l}\right)$ is an $l$-adic Griffiths group.

If $k$ is the algebraic closure of a finite field and $X$ is "of abelian type" and satisfies the Tate conjecture, $\operatorname{Griff}^{2}\left(X, \mathbb{Z}_{l}\right)$ is torsion and $H^{0}\left(X, \mathcal{H}_{\mathrm{ett}}^{3}\left(\mathbb{Q}_{l} / \mathbb{Z}_{l}(2)\right)\right)$ is finite provided $H_{\mathrm{tr}}^{3}\left(X, \mathbb{Q}_{l}(2)\right)=0$. On the other hand, a theorem of Schoen gives an example where $H^{0}\left(X, \mathscr{H}_{\mathrm{et}}^{3}\left(\mathbb{Q}_{l} / \mathbb{Z}_{l}(2)\right)\right)$ is finite but $H_{\mathrm{tr}}^{3}\left(X, \mathbb{Q}_{l}(2)\right) \neq 0$.

\section{Introduction}

Soient $k$ un corps, $X$ une $k$-variété lisse et $l$ un nombre premier différent de car $k$. Uwe Jannsen [1988, Lemma 6.14] a défini une classe de cycle $l$-adique

$$
C H^{2}(X) \otimes \mathbb{Z}_{l} \stackrel{\mathrm{cl}^{2}}{\longrightarrow} H_{\text {cont }}^{4}\left(X, \mathbb{Z}_{l}(2)\right)
$$

à valeurs dans sa cohomologie étale continue. En imitant sa construction à partir d'un théorème de Geisser et Levine [2000], on obtient une variante $p$-adique de (1-1) si $k$ est de caractéristique $p>0$, où le second membre est une version continue de la cohomologie de Hodge-Witt logarithmique. Notons $\mathscr{H}_{\text {ét }}^{3}\left(\mathbb{Q}_{l} / \mathbb{Z}_{l}(2)\right.$ ) (resp. $\left.\mathscr{H}_{\text {cont }}^{3}\left(\mathbb{Z}_{l}(2)\right)\right)$ le faisceau Zariski associé au préfaisceau $U \mapsto H_{\text {ét }}^{3}\left(U, \mathbb{Q}_{l} / \mathbb{Z}_{l}(2)\right)$ (resp. $U \mapsto H_{\text {cont }}^{3}\left(U, \mathbb{Z}_{l}(2)\right)$ ). Le but de cet article est de démontrer l'analogue $l$-adique d'un théorème de Jean-Louis Colliot-Thélène et Claire Voisin [2010, théorème 3.6] :

Théorème 1.1. Soit l un nombre premier quelconque et soit C le conoyau de (1-1). On a une suite exacte

$$
0 \rightarrow H^{0}\left(X, \mathscr{H}_{\text {cont }}^{3}\left(\mathbb{Z}_{l}(2)\right)\right) \otimes \mathbb{Q} / \mathbb{Z} \stackrel{f}{\longrightarrow} H^{0}\left(X, \mathscr{H}_{\text {ét }}^{3}\left(\mathbb{Q}_{l} / \mathbb{Z}_{l}(2)\right)\right) \stackrel{g}{\longrightarrow} C_{\text {tors }} \rightarrow 0 .
$$

Noter que $C_{\text {tors }}$ est fini si $H_{\text {êt }}^{4}\left(X, \mathbb{Z}_{l}(2)\right)$ est un $\mathbb{Z}_{l}$-module de type fini : ceci 
se produit pour $l \neq p$ si $k$ est fini, ou plus généralement si les groupes de cohomologie galoisienne de $k$ à coefficients finis sont finis, par exemple (comme me l'a fait remarquer J.-L. Colliot-Thélène) si $k$ est un corps local [supérieur] ou un corps séparablement clos. Dans ces cas, le théorème 1.1 implique donc que $H^{0}\left(X, \mathscr{H}_{\text {êt }}^{3}\left(\mathbb{Q}_{l} / \mathbb{Z}_{l}(2)\right)\right)$ est extension d'un groupe fini par un groupe divisible. (Pour $l=p, C_{\text {tors }}$ est fini si $k$ est fini et $X$ projective d'après [Gros et Suwa 1988a, p. 589, proposition 4.18].)

Lorsque $k=\mathbb{C}$, ceci donne une autre démonstration du théorème de ColliotThélène-Voisin en utilisant l'isomorphisme de comparaison entre cohomologies de Betti et $l$-adique et sa compatibilité aux classes de cycles respectives.

La démonstration de Colliot-Thélène et Voisin utilise l'exactitude du complexe de faisceaux Zariski de cohomologie de Betti

$$
0 \rightarrow \mathscr{H}_{\text {cont }}^{3}(\mathbb{Z}(2)) \rightarrow \mathscr{H}_{\text {cont }}^{3}(\mathbb{Q}(2)) \rightarrow \mathscr{H}_{\text {êt }}^{3}(\mathbb{Q} / \mathbb{Z}(2)) \rightarrow 0 .
$$

Son exactitude à gauche découle du théorème de Merkurjev-Suslin, c'est-à-dire la conjecture de Bloch-Kato en degré 2 ; celle à droite découle de la conjecture de Bloch-Kato en degré 3, dont la démonstration a été conclue récemment par Voevodsky et un certain nombre d'auteurs.

La démonstration donnée ici évite le recours à cette dernière conjecture : elle ne repose que sur le théorème de Merkurjev-Suslin plus un formalisme triangulé un peu sophistiqué, mais dont, je pense, la sophistication est bien inférieure aux ingrédients de la preuve du théorème de Voevodsky et al.

Son principe est le suivant. La classe de cycle (1-1) se prolonge en une classe «étale»

$$
H_{\text {êt }}^{4}(X, \mathbb{Z}(2)) \otimes \mathbb{Z}_{l} \rightarrow H_{\text {êt }}^{4}\left(X, \mathbb{Z}_{l}(2)\right)
$$

où le terme de gauche est un groupe de cohomologie motivique étale de $X$. Le théorème de comparaison de la cohomologie motivique étale à coefficients finis avec la cohomologie étale des racines de l'unité tordues ou de Hodge-Witt logarithmique (théorème $2.6 \mathrm{a}$ ) et b)) implique que (1-2) est de noyau divisible et de conoyau sans torsion. On en déduit une surjection $g$ de noyau divisible dans le théorème 1.1 à l'aide de la suite exacte

$$
0 \rightarrow C H^{2}(X) \rightarrow H_{\mathrm{e} t}^{4}(X, \mathbb{Z}(2)) \rightarrow H^{0}\left(X, \mathscr{H}_{\mathrm{e} t}^{3}(\mathbb{Q} / \mathbb{Z}(2))\right) \rightarrow 0,
$$

qui est rappelée/établie dans la proposition 2.9. Ceci est fait au §3B. La détermination du noyau est plus technique et je renvoie au §3F pour les détails.

Pour justifier la structure du noyau et du conoyau de (1-2), il faut considérer le « cône » de l'application classe de cycle : ceci est expliqué au §3A.

On obtient de plus des renseignements supplémentaires sur le groupe

$$
H^{0}\left(X, \mathscr{H}_{\text {cont }}^{3}\left(\mathbb{Z}_{l}(2)\right)\right) \otimes \mathbb{Q} / \mathbb{Z}:
$$


1. Si $k$ est fini et $X$ projective lisse, dans la classe $B_{\text {Tate }}(k)$ de [Kahn 2003, définition $1 \mathrm{~b})$ ] ce groupe est nul ( $\$ 5 \mathrm{~A})$.

2. Si $k$ est séparablement clos et $l \neq$ car $k$, toujours dans l'esprit de [Colliot-Thélène et Voisin 2010] on a une suite exacte (corollaire 4.7) :

$$
0 \rightarrow H_{\mathrm{tr}}^{3}\left(X, \mathbb{Z}_{l}(2)\right) \rightarrow H^{0}\left(X, \mathscr{H}_{\mathrm{cont}}^{3}\left(\mathbb{Z}_{l}(2)\right)\right) \rightarrow \operatorname{Griff}^{2}\left(X, \mathbb{Z}_{l}\right) \rightarrow 0
$$

où $\operatorname{Griff}^{2}\left(X, \mathbb{Z}_{l}\right)$ est le groupe des cycles de codimension 2 à coefficients $l$-adiques, modulo l'équivalence algébrique, dont la classe de cohomologie $l$-adique est nulle, et $H_{\text {tr }}^{3}\left(X, \mathbb{Z}_{l}(2)\right)$ est le quotient de $H_{\text {cont }}^{3}\left(X, \mathbb{Z}_{l}(2)\right)$ par le premier cran de la filtration par le coniveau.

Comme le groupe $H^{0}\left(X, \mathscr{H}_{\text {cont }}^{3}\left(\mathbb{Z}_{l}(2)\right)\right)$ est sans torsion (lemme 3.12), on en déduit une suite exacte (proposition 4.12) :

$$
\begin{aligned}
0 \rightarrow \operatorname{Griff}^{2}\left(X, \mathbb{Z}_{l}\right)_{\mathrm{tors}} \rightarrow H_{\mathrm{tr}}^{3}\left(X, \mathbb{Z}_{l}(2)\right) \otimes \mathbb{Q} / \mathbb{Z} \\
\quad \rightarrow H^{0}\left(X, \mathscr{H}_{\mathrm{cont}}^{3}\left(\mathbb{Z}_{l}(2)\right)\right) \otimes \mathbb{Q} / \mathbb{Z} \rightarrow \operatorname{Griff}^{2}\left(X, \mathbb{Z}_{l}\right) \otimes \mathbb{Q} / \mathbb{Z} \rightarrow 0 .
\end{aligned}
$$

3. Si $k$ est la clôture algébrique d'un corps fini $k_{0}$ et que $X$ provient de la classe $B_{\text {Tate }}\left(k_{0}\right)$ de [Kahn 2003], le groupe $\operatorname{Griff}^{2}\left(X, \mathbb{Z}_{l}\right)$ est de torsion et la suite exacte ci-dessus se simplifie en :

$$
0 \rightarrow \operatorname{Griff}^{2}\left(X, \mathbb{Z}_{l}\right) \rightarrow H_{\mathrm{tr}}^{3}\left(X, \mathbb{Z}_{l}(2)\right) \otimes \mathbb{Q} / \mathbb{Z} \rightarrow H^{0}\left(X, \mathscr{H}_{\mathrm{cont}}^{3}\left(\mathbb{Z}_{l}(2)\right)\right) \otimes \mathbb{Q} / \mathbb{Z} \rightarrow 0
$$

(théorème 5.2).

En particulier, $H^{0}\left(X, \mathscr{H}_{\mathrm{ett}}^{3}\left(\mathbb{Q}_{l} / \mathbb{Z}_{l}(2)\right)\right)$ est fini dès que $H_{\mathrm{tr}}^{3}\left(X, \mathbb{Q}_{l}(2)\right)=0$ (ceci est conjecturalement vrai sur tout corps séparablement clos, cf. remarque 4.13), mais un exemple de Schoen montre que cette condition n'est pas nécessaire (proposition 5.5 et théorème 5.6).

\section{Groupes de Chow supérieurs}

Cette section comporte presque exclusivement des rappels sur les groupes de Chow supérieurs : le lecteur au courant peut la parcourir rapidement. Elle a pour but principal de fournir une preuve complète de la proposition 2.9, évitant le complexe $\Gamma(2)$ de Lichtenbaum.

2A. Groupes de Chow supérieurs. Soit $k$ un corps. Bloch [1986] associe à tout $k$-schéma algébrique $X$ des complexes de groupes abéliens $z^{n}(X, \cdot)(n \geq 0)$, concentrés en degrés (homologiques) $\geq 0$ : rappelons qu'on pose

$$
\Delta^{p}=\operatorname{Spec} k\left[t_{0}, \ldots, t_{p}\right] /\left(\sum t_{i}-1\right),
$$


que $z^{n}(X, p)$ est le groupe abélien libre sur les fermés intègres de codimension $n$ de $X \times_{k} \Delta^{p}$ qui rencontrent les faces proprement, et que la différentielle $d_{p}$ est obtenue comme somme alternée des intersections avec les faces de dimension $p-1$. Les groupes d'homologie $C H^{n}(X, p)$ de $z^{n}(X, \bullet)$ sont les groupes de Chow supérieurs de $X$ : on a $C H^{n}(X, 0)=C H^{n}(X)$ par construction.

Les $z^{n}(X, \bullet)$ sont contravariants pour les morphismes plats, en particulier étales ; ils définissent en fait des complexes de faisceaux sur le petit site étale d'un schéma lisse $X$ donné. Ils sont aussi covariants pour les morphismes propres, en particulier pour les immersions fermées.

Si $Y$ est un fermé de $X$, on notera ici

$$
\begin{aligned}
z_{Y}^{n}(X, \cdot) & =\operatorname{Fib}\left(z^{n}(X, \cdot) \stackrel{j^{*}}{\longrightarrow} z^{n}(X-Y, \cdot)\right), \\
C H_{Y}^{n}(X, p) & =H_{p}\left(z_{Y}^{n}(X, \cdot)\right),
\end{aligned}
$$

où $j: X-Y \rightarrow X$ est l'immersion ouverte complémentaire et Fib désigne la fibre homotopique (décalé du mapping cone). Si on tensorise par un groupe abélien $A$, on écrit $\mathrm{CH}_{Y}^{n}(X, p, A)$.

On a le théorème fondamental suivant, qui est une vaste généralisation du lemme de déplacement de Chow ([Bloch 1986, théorèmes 3.1 et 4.1], preuves corrigées dans [Bloch 1994]) :

Théorème 2.1. a) Les groupes de Chow supérieurs sont contravariants pour les morphismes quelconques de but lisse entre variétés quasi-projectives. Ils commutent aux limites projectives filtrantes à morphismes de transition affines.

b) Soient $X$ un $k$-schéma quasi-projectif équidimensionnel, $i: Y \rightarrow X$ un fermé équidimensionnel et $j: U \rightarrow X$ l'ouvert complémentaire. Soit d la codimension de $Z$ dans $X$. Alors le morphisme naturel

$$
z^{n-d}(Y, \cdot) \stackrel{i_{*}}{\longrightarrow} z_{Y}^{n}(X, \cdot)
$$

est un quasi-isomorphisme.

c) On dispose de produits d'intersection

$$
C H^{m}(X, p) \times C H^{n}(X, q) \rightarrow C H^{m+n}(X, p+q)
$$

pour X quasi-projectif lisse.

De la partie b) de ce théorème, on déduit que pour tout groupe abélien $A$, la théorie cohomologique à supports

$$
(X, Z) \mapsto C H_{Z}^{n}(X, \cdot, A)
$$


définie sur la catégorie des $k$-schémas quasi-projectifs lisses [Colliot-Thélène et al. 1997, Definition 5.1.1 a)] vérifie l'axiome COH1 de loc. cit., p. 53. D'après loc. cit., théorème 7.5.2, on a donc des isomorphismes pour tout $(n, p)$

$$
C H^{n}(X, p, A) \stackrel{\sim}{\rightarrow} H_{\mathrm{Zar}}^{-p}\left(X, z^{n}(-, \cdot) \otimes A\right) \stackrel{\sim}{\longrightarrow} H_{\mathrm{Nis}}^{-p}\left(X, z^{n}(-, \cdot) \otimes A\right)
$$

pour $X$ quasi-projectif lisse.

Si $X$ est seulement lisse, le second isomorphisme persiste.

Définition 2.2. Soit $X$ un $k$-schéma lisse (essentiellement de type fini), et soit $\tau$ une topologie de Grothendieck moins fine que la topologie étale sur la catégorie des $k$-schémas lisses de type fini : en pratique $\tau \in\{Z$ Zar, Nis, ét $\}$. On note $A_{X}(n)_{\tau}$ le complexe de faisceaux $z^{n}(-, \cdot) \otimes A[-2 n]$ sur $X_{\tau}$ et $H_{\tau}^{*}(X, A(n))$ l'hypercohomologie de $X$ à coefficients dans le complexe $A_{X}(n)$ (pour la topologie $\tau$ ). C'est la cohomologie motivique de poids $n$ à coefficients dans A pour la topologie concernée.

Pour simplifier, on supprime $\tau$ de la notation si $\tau=$ Zar ou Nis (voir ci-dessus).

On a donc un isomorphisme, pour $X$ quasi-projectif lisse :

$$
C H^{n}(X, 2 n-i) \stackrel{\sim}{\longrightarrow} H^{i}(X, A(n)) .
$$

On a

$$
\begin{aligned}
& \mathbb{Z}_{X}(0)=\mathbb{Z}, \\
& \mathbb{Z}_{X}(1) \simeq 0_{X}^{*}[-1] .
\end{aligned}
$$

([Bloch 1986, corollaire 6.4] pour le second quasi-isomorphisme).

L’isomorphisme (2-4) se généralise ainsi :

Théorème 2.3 [Nesterenko et Suslin 1989; Totaro 1992]. Supposons que $X=$ Spec $K$, où $K$ est un corps. L'isomorphisme (2-4) et les produits (2-1) induisent des isomorphismes

$$
\begin{aligned}
& K_{n}^{M}(K) \stackrel{\sim}{\longrightarrow} H^{n}(K, \mathbb{Z}(n)), \\
& K_{n}^{M}(K) / m \stackrel{\sim}{\longrightarrow} H^{n}(K, \mathbb{Z} / m(n)),
\end{aligned}
$$

pour $m>0$, où $K_{n}^{M}$ désigne la $K$-théorie de Milnor.

Démonstration. Voir les travaux cités pour le premier énoncé ; le second s'en déduit puisque $H^{n+1}(K, \mathbb{Z}(n))=0$.

Remarque 2.4. L'isomorphisme (2-2) vaut pour $i \geq 2 n$, même si $X$ n'est pas quasiprojectif. En effet, le terme de droite est l'aboutissement d'une suite spectrale de coniveau [Colliot-Thélène et al. 1997, Remark 5.1.3 (3)] qui, grâce au théorème 2.1, 
prend la forme suivante :

$$
E_{1}^{p, q}=\bigoplus_{x \in X^{(p)}} H^{q-p}(k(x), A(n-p)) \Rightarrow H^{p+q}(X, A(n)) .
$$

On a $H^{i}(F, A(r))=0$ pour $i>r$ et tout corps $F$, car $A_{F}(r)$ est un complexe concentré en degrés $\leq r$. On en déduit déjà que $H^{i}(X, A(n))=0$ pour $i>2 n$, et on a évidemment $C H^{n}(X, p, A)=0$ pour $p<0$. Quant à $H^{2 n}(X, A(n))$, il s'insère dans une suite exacte

$$
E_{1}^{n-1, n} \stackrel{d_{1}}{\longrightarrow} E_{1}^{n, n} \rightarrow H^{2 n}(X, A(n)) \rightarrow 0
$$

qui s'identifie à la suite exacte

$$
\bigoplus_{x \in X_{(1)}} k(x)^{*} \otimes A \stackrel{\text { Div }}{\longrightarrow} \bigoplus_{x \in X_{(0)}} A \rightarrow C H^{n}(X) \otimes A \rightarrow 0
$$

via (2-3) et (2-4) (l'identification de la différentielle $d_{1}$ à l'application diviseurs est facile à partir du théorème 2.1 appliqué pour $n=1$ ). D'autre part, on calcule aisément que $C H^{n}(X, 0, A)=C H^{n}(X) \otimes A$ sans supposer $X$ quasi-projectif.

Le lemme suivant raffine une partie de la remarque 2.4 : sa démonstration est moins élémentaire.

Lemme 2.5. Le complexe $\mathbb{Z}_{X}(n)$ est concentré en degrés $\leq n$ : autrement dit, $\mathscr{H}^{i}\left(\mathbb{Z}_{X}(n)\right)=0$ pour $i>n$.

Démonstration. La théorie cohomologique à supports

$$
(X, Y) \mapsto H_{Y}^{*}(X, \mathbb{Z}(n))
$$

vérifie les axiomes $\mathrm{COH} 1$ et $\mathrm{COH} 3$ de [Colliot-Thélène et al. 1997] : le premier, « excision étale », résulte facilement du théorème 2.1 b) et le second, invariance par homotopie, est démontré dans [Bloch 1986, théorème 2.1]. Il résulte alors de [Colliot-Thélène et al. 1997, Corollary 5.1.11] qu'elle vérifie la conjecture de Gersten (c'est déjà démontré dans [Bloch 1986, théorème 10.1]). En particulier, les faisceaux $\mathscr{H}^{i}(\mathbb{Z}(n))$ s'injectent dans leur fibre générique, et on est réduit au cas évident d'un corps de base.

2B. Comparaisons. À partir de maintenant, $X$ désigne un $k$-schéma lisse.

Théorème 2.6. a) Si $m$ est inversible dans $k$, il existe un quasi-isomorphisme canonique

$$
(\mathbb{Z} / m)_{X}(n)_{\text {ét }}^{\stackrel{\sim}{\longrightarrow}} \mu_{m}^{\otimes n}
$$


b) Si $k$ est de caractéristique $p>0$ et $r \geq 1$, il existe un quasi-isomorphisme canonique

$$
\left(\mathbb{Z} / p^{r}\right)_{X}(n)_{\text {ét }}^{\stackrel{\sim}{\longrightarrow}} v_{r}(n)[-n]
$$

où $v_{r}(n)$ est le n-ème faisceau de Hodge-Witt logarithmique.

c) Soit $\alpha$ la projection de $X_{\text {ét }}$ sur $X_{\mathrm{Zar}}$. Alors la flèche d'adjonction

$$
\mathbb{Q}_{X}(n) \rightarrow R \alpha_{*} \mathbb{Q}_{X}(n)_{\text {ét }}
$$

est un isomorphisme.

Démonstration. a) et b) sont dus à Geisser-Levine : a) est [2001, Theorem 1.5] et b) est [2000, Theorem 8.5] (si $k$ est parfait : voir le corollaire A.7 en général). ${ }^{1}$ Quant à c), c'est un fait général pour un complexe de faisceaux Zariski $C$ de $\mathbb{Q}$-espaces vectoriels sur un schéma normal $S:$ on se ramène au cas où $S$ est local et où $C=A[0]$ est un faisceau concentré en degré 0 . Alors $A$ est un faisceau constant de $\mathbb{Q}$-espaces vectoriels et cela résulte de [Deninger 1988, Theorem 2.1].

Définition 2.7. Soit $n \geq 0$. Pour $l$ premier différent de car $k$, on note

$$
\mathbb{Q}_{l} / \mathbb{Z}_{l}(n)=\underset{r}{\lim } \mu_{l^{r}}^{\otimes n}
$$

Pour $l=$ car $k$, on note

$$
\mathbb{Q}_{l} / \mathbb{Z}_{l}(n)=\underset{r}{\lim } v_{r}(n)[-n]
$$

Enfin, on note

$$
\mathbb{Q} / \mathbb{Z}(n)=\bigoplus_{l} \mathbb{Q}_{l} / \mathbb{Z}_{l}(n)
$$

C'est un objet de la catégorie dérivée des groupes abéliens sur le gros site étale de Spec $k$.

Le théorème 2.6 montre qu'on a un isomorphisme, pour tout $X$ lisse sur $k$ :

$$
(\mathbb{Q} / \mathbb{Z})_{X}(n)_{\text {ét }} \stackrel{\sim}{\longrightarrow}(\mathbb{Q} / \mathbb{Z})(n)_{\mid X} .
$$

Nous utiliserons cette identification dans la suite sans mention ultérieure.

On a alors :

Corollaire 2.8. Pour tout $i>n+1$, l'homomorphisme de faisceaux Zariski

$$
\mathscr{H} \mathcal{H}^{i-1}\left(R \alpha_{*} \mathbb{Q} / \mathbb{Z}(n)\right) \rightarrow \mathscr{H}^{i}\left(R \alpha_{*} \mathbb{Z}_{X}(n)_{\text {ét }}\right)
$$

émanant du théorème 2.6 a) et b) est un isomorphisme.

1. Cette dernière référence indique que l'hypothèse « $X$ lisse sur $k »$ devrait être remplacée par « $X$ régulier de type fini sur $k »$ dans une grande partie de ce texte. 
Démonstration. Dans la suite exacte de faisceaux Zariski

$\mathscr{H}^{i-1}\left(R \alpha_{*} \mathbb{Q}_{X}(n)_{\text {ét }}\right) \rightarrow \mathscr{H}^{i-1}\left(R \alpha_{*} \mathbb{Q} / \mathbb{Z}(n)\right) \rightarrow \mathscr{H}^{i}\left(R \alpha_{*} \mathbb{Z}_{X}(n)_{\text {ét }}\right) \rightarrow \mathscr{H}^{i}\left(R \alpha_{*} \mathbb{Q}_{X}(n)_{\text {ét }}\right)$

les deux termes extrêmes sont nuls d'après le théorème $2.6 \mathrm{c}$ ) et le lemme 2.5.

2C. Cohomologie étale de complexes non bornés. Si $X$ est un schéma de dimension cohomologique étale $a$ priori non finie et si $C$ est un complexe de faisceaux étales sur $X$, non borné inférieurement, la considération de l'hypercohomologie $H_{\text {êt }}^{*}(X, C)$ soulève au moins trois difficultés : 1) une définition en forme; 2) la commutation aux limites ; 3) la convergence de la suite spectrale d'hypercohomologie.

Le premier problème est maintenant bien compris : il faut prendre une résolution K-injective, ou fibrante, de $C$; voir par exemple [Spaltenstein 1988, Theorem 4.5 et Remark 4.6].

Le second et le troisième problèmes sont plus délicats. Dans le cas de $\mathbb{Z}(n)$, le second et implicitement le troisième est résolu dans [Kahn 1997, §B.3 p. 1114] (pour la cohomologie motivique de Suslin-Voevodsky). Rappelons l'argument : en utilisant le théorème 2.6, on peut insérer $R \alpha_{*} \mathbb{Z}_{X}(n)$ ét dans un triangle exact

$$
R \alpha_{*} \mathbb{Z}_{X}(n)_{\text {ét }} \rightarrow \mathbb{Q}_{X}(n) \rightarrow R \alpha_{*}(\mathbb{Q} / \mathbb{Z})_{X}(n)_{\text {ét }} \stackrel{+1}{\longrightarrow} \text {. }
$$

Si $X$ est de dimension de Krull finie, l'hypercohomologie Zariski du second terme se comporte bien, et celle du troisième terme aussi puisque c'est l'hypercohomologie étale d'un complexe borné.

2D. Conjecture de Beilinson-Lichtenbaum. Cette conjecture concerne la comparaison entre $H^{*}(X, A(n))$ et $H_{\mathrm{ett}}^{*}(X, A(n))$, pour $A=\mathbb{Z} / m$, cf. [Geisser et Levine 2001, Theorem 1.6]. Si $m$ est une puissance d'un nombre premier $l \neq \operatorname{car} k$, elle est équivalente d'après [Geisser et Levine 2001] à la conjecture de Bloch-Kato en poids $n$ (pour le nombre premier $l$ ); donc en poids 2, au théorème de Merkurjev-Suslin. Sur un corps de caractéristique zéro, ceci avait été antérieurement démontré dans [Suslin et Voevodsky 2000].

De plus, pour $l=$ car $k$, une version de cette conjecture est démontrée par Geisser et Levine [2000] ; voir théorème A.5. En ajoutant à tout ceci le théorème 2.6 c), la conjecture de Beilinson-Lichtenbaum en poids $n$ se retraduit en un triangle exact [Voevodsky 2003, Theorem 6.6]

$$
\mathbb{Z}_{X}(n) \rightarrow R \alpha_{*} \mathbb{Z}_{X}(n)_{\text {ét }} \rightarrow \tau_{\geq n+2} R \alpha_{*} \mathbb{Z}_{X}(n)_{\text {ét }} \rightarrow \mathbb{Z}_{X}(n)[1] .
$$

Ce triangle exact contient l'énoncé (« Hilbert 90 en poids $n »)$ :

$$
\mathscr{H}^{n+1}\left(R \alpha_{*} \mathbb{Z}_{X}(n)_{\text {ét }}\right)=0 .
$$




\section{E. Une suite exacte.}

Proposition 2.9. Notons $\mathscr{H}_{\mathrm{ett}}^{3}(\mathbb{Q} / \mathbb{Z}(2))$ le faisceau Zariski associé au préfaisceau $U \mapsto H_{\mathrm{et}}^{3}(U, \mathbb{Q} / \mathbb{Z}(2))$. Pour toute $k$-variété lisse $X$, on a une suite exacte courte:

$$
0 \rightarrow C H^{2}(X) \rightarrow H_{\text {êt }}^{4}(X, \mathbb{Z}(2)) \rightarrow H^{0}\left(X, \mathscr{H}_{\text {êt }}^{3}(\mathbb{Q} / \mathbb{Z}(2))\right) \rightarrow 0 .
$$

Démonstration. En prenant l'hypercohomologie de Zariski de $X$ à valeurs dans le triangle (2-6) pour $n=2$, on trouve une suite exacte

$$
0 \rightarrow H^{4}(X, \mathbb{Z}(2)) \rightarrow H_{\text {êt }}^{4}(X, \mathbb{Z}(2)) \rightarrow H^{0}\left(X, R^{4} \alpha_{*} \mathbb{Z}(2) \text { ét }\right) \rightarrow H^{5}(X, \mathbb{Z}(2)) \text {. }
$$

D'après la remarque 2.4 , on a $H^{4}(X, \mathbb{Z}(2))=C H^{2}(X)$ et $H^{5}(X, \mathbb{Z}(2))=0$. D'autre part, le triangle exact

$$
\mathbb{Z}_{X}(2)_{\text {ét }} \rightarrow \mathbb{Q}_{X}(2)_{\text {ét }} \rightarrow(\mathbb{Q} / \mathbb{Z})_{X}(2)_{\text {ét }}
$$

provenant du théorème 2.6 donne une longue suite exacte de faisceaux

$$
R^{3} \alpha_{*} \mathbb{Q}_{X}(2)_{\text {ét }} \rightarrow R^{3} \alpha_{*}(\mathbb{Q} / \mathbb{Z})_{X}(2)_{\text {ét }} \rightarrow R^{4} \alpha_{*} \mathbb{Z}_{X}(2)_{\text {ét }} \rightarrow R^{4} \alpha_{*} \mathbb{Q}_{X}(2)_{\text {ét }} .
$$

On a $R^{3} \alpha_{*} \mathbb{Q}_{X}(2)_{\text {ét }}=R^{4} \alpha_{*} \mathbb{Q}_{X}(2)$ ét $=0$ puisque $\mathbb{Z}_{X}(2)$ est concentré en degrés $\leq 2$, d'après le théorème $2.6 \mathrm{c}$ ). Ce qui conclut, via l'isomorphisme (2-5).

Remarques 2.10. 1) La suite exacte (2-7) apparaît dans [Kahn 1996, théorème 1.1, équation (9)], avec $\mathbb{Z}(2)$ remplacé par le complexe de Lichtenbaum $\Gamma(2)$; à la 2torsion près, elle est déjà chez Lichtenbaum [1990, Theorem 2.13 et Remark 2.14]. Il est probable qu'on a un isomorphisme

$$
\Gamma(2, X) \simeq \tau_{\geq 1}\left(z^{2}(X, \cdot)[-4]\right)
$$

dans $D\left(X_{\text {Zar }}\right)$ pour tout $k$-schéma lisse $X .{ }^{2}$ Une fonctorialité suffisante de cet isomorphisme impliquerait qu'il peut s'étalifier. Dans [Block 1995, Theorem 7.2], un isomorphisme (2-8) est construit pour $X=\operatorname{Spec} k$. Mais (2-8) ne semble pas apparaître dans la littérature en général.

2) En se reposant sur la conjecture de Bloch-Kato en poids 3, on obtient de la même manière une suite exacte

$$
\begin{aligned}
0 \rightarrow H^{2}\left(X, \mathscr{K}_{3}\right) \rightarrow H_{\mathrm{et}}^{5}(X, \mathbb{Z}(3)) \rightarrow H^{0}\left(X, \mathscr{H}_{\mathrm{et}}^{4}(\mathbb{Q} / \mathbb{Z}(3))\right. & \\
& \rightarrow C H^{3}(X) \rightarrow H_{\mathrm{èt}}^{6}(X, \mathbb{Z}(3)) .
\end{aligned}
$$

Cette suite apparait dans [Kahn 2003, remarque 4.10], sauf que le premier terme est $H^{5}(X, \mathbb{Z}(3))$; son identification avec $H^{2}\left(X, \mathscr{K}_{3}\right)$ se fait à l'aide de la suite spectrale de coniveau de la remarque 2.4 .

2. Par ailleurs, la conjecture de Beilinson-Soulé prédit que $\mathbb{Z}(2) \rightarrow \tau_{\geq 1} \mathbb{Z}(2)$ est un quasiisomorphisme, mais elle n'a pas d'importance pour ce travail. 


\section{F. D'autres suites exactes.}

Proposition 2.11. On a des suites exactes

$$
\begin{aligned}
0 \rightarrow H^{3}(X, \mathbb{Z} / m(2)) \rightarrow H_{\text {êt }}^{3}(X, \mathbb{Z} / m(2)) & \rightarrow H^{0}\left(X, \mathscr{H}_{\text {êt }}^{3}(\mathbb{Z} / m(2))\right) \\
& \rightarrow C H^{2}(X) \otimes \mathbb{Z} / m \rightarrow H_{\text {êt }}^{4}(X, \mathbb{Z} / m(2))
\end{aligned}
$$

$(m>0)$,

$$
\begin{aligned}
0 \rightarrow H^{3}(X, \mathbb{Q} / \mathbb{Z}(2)) \rightarrow H_{\text {êt }}^{3}(X, \mathbb{Q} / \mathbb{Z}(2)) & \rightarrow H^{0}\left(X, \mathscr{H}_{\text {êt }}^{3}(\mathbb{Q} / \mathbb{Z}(2))\right) \\
& \rightarrow C H^{2}(X) \otimes \mathbb{Q} / \mathbb{Z} \rightarrow H_{\text {êt }}^{4}(X, \mathbb{Q} / \mathbb{Z}(2)) .
\end{aligned}
$$

Démonstration. Elles s'obtiennent comme dans la preuve de la proposition 2.9 en prenant la cohomologie des triangles exacts

$$
\begin{aligned}
& \mathbb{Z} / m(2) \rightarrow R \alpha_{*}(\mathbb{Z} / m)_{\text {ét }}(2) \rightarrow \tau_{\geq 3} R \alpha_{*}(\mathbb{Z} / m)_{\text {ét }}(2) \stackrel{+1}{\longrightarrow} \\
& \mathbb{Q} / \mathbb{Z}(2) \rightarrow R \alpha_{*}(\mathbb{Q} / \mathbb{Z})_{\text {ét }}(2) \rightarrow \tau_{\geq 3} R \alpha_{*}(\mathbb{Q} / \mathbb{Z})_{\text {ét }}(2) \stackrel{+1}{\longrightarrow}
\end{aligned}
$$

obtenus en tensorisant (2-6) par $\mathbb{Z} / m$ ou $\mathbb{Q} / \mathbb{Z}$ au sens dérivé.

On reconnaît donc dans $H^{3}(X, \mathbb{Z} / m(2))$ le groupe $N H_{\text {êt }}^{3}(X, \mathbb{Z} / m(2))$ de Suslin [1987, §4]. On peut sans doute montrer que la seconde suite exacte coïncide avec celle de [Colliot-Thélène et al. 1983, p. 790, Remark 2].

\section{Cohomologie $l$-adique et $p$-adique}

Dans cette section, $k$ est un corps quelconque, de caractéristique $p \geq 0$.

3A. Classe de cycle l-adique et p-adique. Soit $l$ un nombre premier quelconque. Pour toute $k$-variété lisse $X$, on a des applications «classe de cycle $l$-adique »

$$
H_{\mathrm{ett}}^{i}(X, \mathbb{Z}(n)) \otimes \mathbb{Z}_{l} \rightarrow H_{\text {cont }}^{i}\left(X, \mathbb{Z}_{l}(n)\right) .
$$

Ces homomorphismes proviennent d'un morphisme de complexes (dans la catégorie dérivée de la catégorie des complexes de faisceaux sur $X_{\text {ét }}$ )

$$
\mathbb{Z}_{X}(n)_{\text {ét }}^{\stackrel{L}{\otimes}} \mathbb{Z}_{l} \rightarrow \mathbb{Z}_{l}(n)_{X}^{c}
$$

où

$$
\mathbb{Z}_{l}(n)_{X}^{c}= \begin{cases}R \lim _{\lim _{l^{r}}}^{\otimes n} & \text { si } l \neq p \\ R \lim _{\longleftarrow} v_{r}(n)[-n] & \text { si } l=p .\end{cases}
$$

Cette construction est décrite dans [Kahn 2002, §1.4, en particulier (1.8)] pour $l \neq p$ et dans [Kahn 2003, §3.5] pour $l=p$. Elle repose sur celles de Geisser-Levine aux crans finis ([2001] pour $l \neq p$ et [2000, démonstration du théorème 8.3] pour $l=p)$. 
Remarque 3.1. Pour $l \neq p$ et $i=2 n$, la composée de (3-1) avec l'homomorphisme $C H^{n}(X) \otimes \mathbb{Z}_{l} \rightarrow H_{\text {ét }}^{2 n}(X, \mathbb{Z}(n)) \otimes \mathbb{Z}_{l}$ (2-7) n'est autre que la classe de cycle de Jannsen [1988, Lemma 6.14] : cela résulte de la construction même dans [Geisser et Levine 2001] de l'isomorphisme du théorème 2.6 a). Pour $l=p$, il est moins clair que (3-1) soit compatible avec la classe de cycle de Gros [1985, p. 50, définition 4.1.7 et p. 55, proposition 4.2.33]. Cela doit pouvoir se vérifier directement ; comme je n'en aurai pas besoin, je laisse cet « exercice »aux lecteurs intéressés.

Notons les isomorphismes évidents :

$$
\mathbb{Z}_{l}(n)_{X}^{c} \stackrel{L}{\otimes} \mathbb{Z} / l^{r} \stackrel{\sim}{\longrightarrow} \begin{cases}\mu_{l^{r}}^{\otimes n} & \text { si } l \neq p \\ v_{r}(n)[-n] & \text { si } l=p .\end{cases}
$$

Définition 3.2. On note respectivement $K_{X}(n)$ ét et $K_{X}(n)$ le choix d'un cône du morphisme (3-2) et du morphisme composé

$$
\mathbb{Z}_{X}(n) \stackrel{L}{\otimes} \mathbb{Z}_{l} \rightarrow R \alpha_{*} \mathbb{Z}_{X}(n)_{\text {ét }}^{\stackrel{L}{\otimes}} \mathbb{Z}_{l} \rightarrow R \alpha_{*} \mathbb{Z}_{l}(n)_{X}^{c}
$$

de sorte qu'on a un morphisme

$$
K_{X}(n) \rightarrow R \alpha_{*} K_{X}(n)_{\text {ét }}
$$

compatible avec le morphisme $\mathbb{Z}_{X}(n) \stackrel{L}{\otimes} \mathbb{Z}_{l} \rightarrow R \alpha_{*} \mathbb{Z}_{X}(n)_{\text {ét }} \stackrel{L}{\otimes} \mathbb{Z}_{l}$.

Remarque 3.3. Rappelons que $K_{X}(n)$ et $K_{X}(n)$ ét ne sont uniques qu'à isomorphisme non unique près; le morphisme $K_{X}(n) \rightarrow R \alpha_{*} K_{X}(n)$ ét n'a pas non plus d'unicité particulière. En particulier, ces choix ne sont fonctoriels en $X$ que pour les immersions ouvertes : cela suffira pour nos besoins ici. Toutefois, on pourrait faire des choix plus rigides (fonctoriels pour les morphismes quelconques entre schémas lisses), quitte à travailler dans des catégories de modèles convenables.

En vertu du théorème 2.6, (3-3) implique immédiatement :

Proposition 3.4. Le morphisme (3-2) $\otimes \mathbb{Z} / l^{r}$ est un isomorphisme pour tout entier $r \geq 1$. Autrement dit, les faisceaux de cohomologie de $K_{X}(n)$ ét sont uniquement l-divisibles.

Corollaire 3.5. Pour tout $(X, i, n)$, le noyau de (3-1) est $l$-divisible et son conoyau est sans l-torsion.

Démonstration. On a une suite exacte

$$
H_{\text {ét }}^{i-1}(X, K(n)) \rightarrow H_{\text {êt }}^{i}(X, \mathbb{Z}(n)) \otimes \mathbb{Z}_{l} \rightarrow H_{\text {cont }}^{i}\left(X, \mathbb{Z}_{l}(n)\right) \rightarrow H_{\text {êt }}^{i}(X, K(n))
$$

(où $H_{\text {êt }}^{*}(X, K(n)):=\mathbb{U}_{\text {êt }}^{*}\left(X, K_{X}(n)\right.$ ét $)$ ), dont les termes extrêmes sont uniquement divisibles. 
3B. Démonstration du théorème 1.1 : première partie. On va démontrer :

Proposition 3.6. Soit $C$ le conoyau de (1-1). On a une surjection

$$
H^{0}\left(X, \mathscr{H}_{\mathrm{et}}^{3}\left(\mathbb{Q}_{l} / \mathbb{Z}_{l}(2)\right)\right) \longrightarrow C_{\text {tors }}
$$

de noyau divisible.

Démonstration. Utilisons la suite exacte (2-7) : en chassant dans le diagramme commutatif de suites exactes

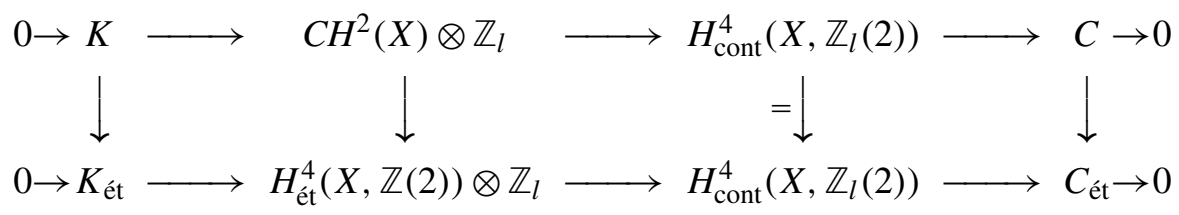

(définissant $K, K_{\text {ét }}, C$ et $C_{\text {ét) }}$ ), on en déduit une suite exacte

$$
0 \rightarrow K \rightarrow K_{\text {ét }} \rightarrow H^{0}\left(X, \mathcal{H}_{\text {êt }}^{3}\left(\mathbb{Q}_{l} / \mathbb{Z}_{l}(2)\right)\right) \rightarrow C \rightarrow C_{\text {ét }} \rightarrow 0 .
$$

On conclut en utilisant le corollaire 3.5.

3C. Suites spectrales de coniveau. Soit $C$ un complexe de faisceaux Zariski sur $X$. Par une technique bien connue remontant à Grothendieck et Hartshorne (cf. [Colliot-Thélène et al. 1997, 1.1]) on obtient une suite spectrale

$$
E_{1}^{p, q}=\bigoplus_{x \in X^{(p)}} H_{x}^{p+q}(X, C) \Rightarrow H^{p+q}(X, C) .
$$

Cette suite spectrale est clairement naturelle en $C \in D\left(X_{\text {Zar }}\right)$. On notera de manière suggestive :

$$
E_{2}^{p, q}=A^{p}\left(X, H^{q}(C)\right)
$$

de sorte qu'on a des morphismes « edge »

$$
H^{n}(X, C) \rightarrow A^{0}\left(X, H^{n}(C)\right)
$$

Lorsque $C$ vérifie la conjecture de Gersten, on a des isomorphismes canoniques

$$
A^{p}\left(X, H^{q}(C)\right) \simeq H^{p}\left(X, \mathscr{H}^{q}(C)\right) .
$$

D'après [Colliot-Thélène et al. 1997, Corollary 5.1.11] c'est le cas pour $C=$ $R \alpha_{*} \mathbb{Z}_{l}(n)_{X}^{c}$. En effet, pour $l \neq p$, la théorie cohomologique à supports correspondante vérifie les axiomes $\mathrm{COH} 1$ (excision étale, on dit maintenant Nisnevich) et COH3 (invariance par homotopie) de [Colliot-Thélène et al. 1997]; pour $l=p$, elle vérifie $\mathrm{COH} 1$ et $\mathrm{COH} 5$. Ce dernier axiome est la «formule du fibré projectif »: il résulte de [Gros 1985]. Si $k$ est un corps fini, il faut adjoindre à ces axiomes l'axiome COH6 de [Colliot-Thélène et al. 1997, p. 64] (existence de transferts pour les extensions finies) : il est standard. 
C'est également le cas pour $C=\mathbb{Z}_{X}(n)$, cf. preuve du lemme 2.5. Par contre ce n'est pas clair pour $C=K_{X}(n)$ : en effet, la règle $(X, Y) \mapsto H_{Y}^{*}(X, K(n))$ ne définit pas une théorie cohomologique à supports sans un choix cohérent des cônes $K_{X}(n)$. Plus précisément, cette règle n'est pas a priori fonctorielle en $(X, Y)$ pour les morphismes quelconques de paires. On ne peut donc pas lui appliquer la théorie de Bloch-Ogus-Gabber développée dans [Colliot-Thélène et al. 1997]. La considération des suites spectrales de coniveau nous permettra de contourner ce problème.

3D. Un encadrement de la cohomologie non ramifiée. L'identification du noyau de (3-4) est plus délicate. À titre préparatoire, on va pousser l'analyse du §3A un peu plus loin en faisant intervenir la conjecture de Bloch-Kato en degré $n$.

Par l'axiome de l'octaèdre (et la conjecture de Bloch-Kato, cf. (2-6)), on a un diagramme commutatif de triangles distingués dans $D\left(X_{\text {Zar }}\right)$, où $K_{X}(n)$ et $K_{X}(n)$ ét ont été introduits dans la définition 3.2 :

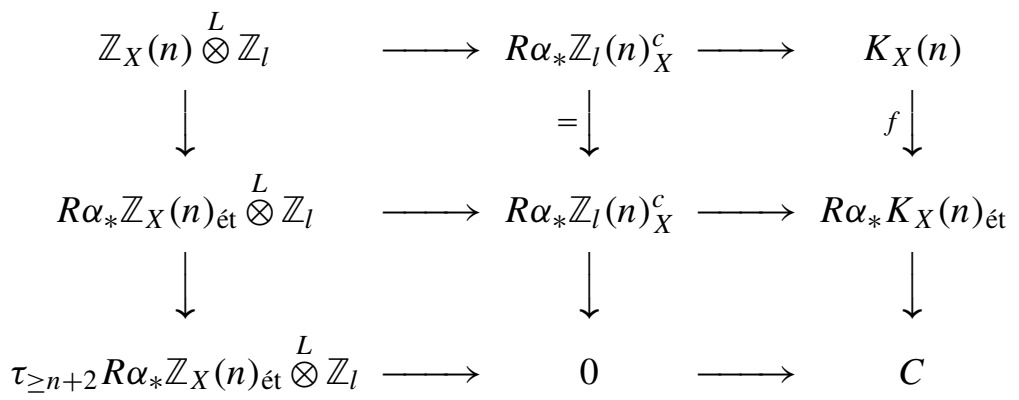

et où $C$ est par définition «le » cône de $f$. On a donc un zig-zag d'isomorphismes

$$
C \stackrel{\sim}{\longrightarrow} \tau_{\geq n+2} R \alpha_{*} \mathbb{Z}_{X}(n)_{\text {ét }} \stackrel{L}{\otimes} \mathbb{Z}_{l}[1] \stackrel{\sim}{\longleftarrow} \tau_{\geq n+1} R \alpha_{*}\left(\mathbb{Q}_{l} / \mathbb{Z}_{l}\right)(n)_{\text {ét }}
$$

où l'isomorphisme de gauche provient du diagramme ci-dessus, et celui de droite provient du corollaire 2.8. D'où un triangle exact

$$
K_{X}(n) \rightarrow R \alpha_{*} K_{X}(n)_{\text {ét }} \rightarrow \tau_{\geq n+1} R \alpha_{*}\left(\mathbb{Q}_{l} / \mathbb{Z}_{l}\right)(n)_{\text {ét }} \stackrel{+1}{\longrightarrow} .
$$

Comme le deuxième terme est uniquement divisible (proposition 3.4) et que le troisième est de torsion, cela montre que

$$
K_{X}(n) \otimes \mathbb{Q} \stackrel{\sim}{\longrightarrow} R \alpha_{*} K_{X}(n) \text { ét. }
$$

On en déduit :

Lemme 3.7. Soit $l \neq p$. Sous la conjecture de Bloch-Kato en degré $n$, le groupe $H^{i}(X, K(n))$ est uniquement divisible pour $i \leq n$ et on a une suite exacte courte $0 \rightarrow H^{n+1}(X, K(n)) \otimes \mathbb{Q} / \mathbb{Z} \rightarrow H^{0}\left(X, \mathscr{H}^{n+1}\left(\mathbb{Q}_{l} / \mathbb{Z}_{l}(n)\right)\right) \rightarrow H^{n+2}(X, K(n))_{\text {tors }} \rightarrow 0$. Le même énoncé vaut pour $l=p$, en utilisant le théorème A.5. 
Le point est maintenant d'identifier les termes extrêmes de la suite du lemme 3.7 à des groupes plus concrets : nous n'y parvenons que pour $n=2$ au $\S 3 \mathrm{~F}$. Mais pour $n$ quelconque, notons la suite exacte

$$
\begin{aligned}
0 \rightarrow \operatorname{Coker}\left(H^{n+2}(X, \mathbb{Z}(n)) \otimes \mathbb{Z}_{l}\right. & \left.\rightarrow H_{\text {cont }}^{n+2}\left(X, \mathbb{Z}_{l}(n)\right)\right) \\
& \rightarrow H^{n+2}(X, K(n)) \rightarrow H^{n+3}(X, \mathbb{Z}(n)) \otimes \mathbb{Z}_{l}
\end{aligned}
$$

et les homomorphismes évidents :

$$
H^{n+1}(X, K(n)) \stackrel{\alpha}{\rightarrow} A^{0}\left(X, H^{n+1}(K(n))\right) \stackrel{\beta}{\leftarrow} A^{0}\left(X, H_{\text {cont }}^{n+1}\left(\mathbb{Z}_{l}(n)\right)\right)
$$

où $\alpha$ est l'homomorphisme (3-6). La suite exacte (3-8) en induit une sur les sousgroupes de torsion. Pour $n=2$, le dernier terme est nul : on retrouve ainsi la proposition 3.6. Pour $n>2$, la première flèche de (3-8) n'a plus de raison d'être surjective sur la torsion. Notons tout de même que pour $n=3$, le dernier terme de (3-8) n'est autre que $C H^{3}(X) \otimes \mathbb{Z}_{l}$ (comparer aux remarques 2.10).

D'autre part :

Lemme 3.8. L'homomorphisme $\beta$ de (3-9) est un isomorphisme pour tout $n \geq 0$.

Démonstration. Dans le diagramme commutatif

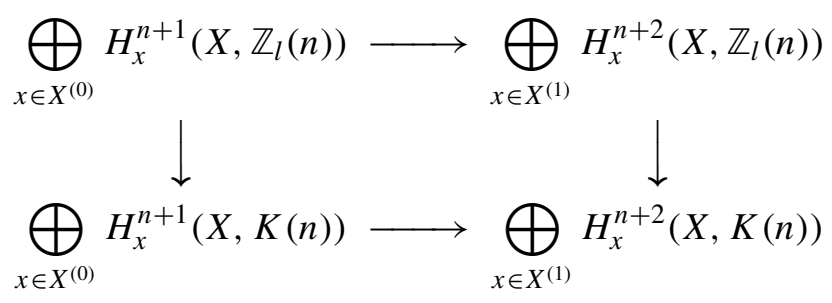

les deux flèches verticales sont des isomorphismes. En effet, elle s'insèrent dans des suites exactes du type

$$
\begin{aligned}
& H_{x}^{n+1}(X, \mathbb{Z}(n)) \otimes \mathbb{Z}_{l} \rightarrow H_{x}^{n+1}\left(X, \mathbb{Z}_{l}(n)\right) \rightarrow H_{x}^{n+1}(X, K(n)) \rightarrow H_{x}^{n+2}(X, \mathbb{Z}(n)) \otimes \mathbb{Z}_{l} \\
& H_{x}^{n+2}(X, \mathbb{Z}(n)) \otimes \mathbb{Z}_{l} \rightarrow H_{x}^{n+2}\left(X, \mathbb{Z}_{l}(n)\right) \rightarrow H_{x}^{n+2}(X, K(n)) \rightarrow H_{x}^{n+3}(X, \mathbb{Z}(n)) \otimes \mathbb{Z}_{l}
\end{aligned}
$$

où dans la première suite, $x$ est de codimension 0 et dans la seconde suite, $x$ est de codimension 1. Sans perte de généralité, on peut supposer $X$ connexe et alors, pour son point générique $\eta$ (cf. théorème $2.1 \mathrm{a})$ ) :

$$
H_{\eta}^{i}(X, \mathbb{Z}(n)):=\underset{U}{\lim } H^{i}(U, \mathbb{Z}(n)) \stackrel{\sim}{\longrightarrow} H^{i}(k(X), \mathbb{Z}(n))=0 \text { pour } i>n .
$$

Pour $x$ de codimension 1 , on a

$$
H_{x}^{i}(X, \mathbb{Z}(n)):=\underset{\lim _{\ni x}}{\log } H_{Z_{U}}^{i}(U, \mathbb{Z}(n))
$$


où $Z_{U}=\overline{\{x\}} \cap U$. Grâce au théorème $2.1 \mathrm{~b}$ ) et a), cette limite devient

$$
\underset{U \ni x}{\lim } H^{i-2}\left(Z_{U}, \mathbb{Z}(n-1)\right)=H^{i-2}(k(x), \mathbb{Z}(n-1))=0 \text { pour } i-2>n-1 .
$$

Le lemme en découle.

3E. Cohomologie à supports de $K(n)$. On aura aussi besoin des deux lemmes suivants au prochain numéro :

Lemme 3.9. Soit $l \neq$ car $k$.

Soit $Y \subset X$ un couple lisse de codimension d. Alors il existe des isomorphismes

$$
H_{\mathrm{Zar}}^{i-2 d}(Y, K(n-d)) \stackrel{\sim}{\longrightarrow} H_{Y}^{i}(X, K(n)) \quad(n \geq 0, i \in \mathbb{Z})
$$

contravariants pour les immersions ouvertes $U \hookrightarrow X$.

Attention : ce lemme affirme l'existence d'isomorphismes de pureté, mais ne dit rien sur leur caractère canonique ou fonctoriel au-delà de la contravariance énoncée. (On peut faire en sorte qu'ils soient contravariants pour les morphismes quelconques entre variétés lisses, mais c'est plus technique et inutile ici.)

Démonstration. Notons $i$ l'immersion fermée $Y \rightarrow X$. On remarque que le diagramme de $D\left(Y_{\mathrm{Zar}}\right)$

$$
\begin{aligned}
& \mathbb{Z}(n-d)_{Y} \otimes \mathbb{Z}_{l}[-2 d] \stackrel{\mathrm{cl}_{Y}^{n-d}}{\longrightarrow} R \alpha_{*} \mathbb{Z}_{l}(n-d)_{Y}^{c}[-2 d] \\
& f \downarrow \\
& R i_{\text {Zar }}^{!} \mathbb{Z}(n)_{X} \otimes \mathbb{Z}_{l} \stackrel{\mathrm{cl}_{X}^{n}}{\longrightarrow} \quad R \alpha_{*} R i_{\text {êt }}^{!} \mathbb{Z}_{l}(n)_{X}^{c}
\end{aligned}
$$

où $f$ est induit par le théorème $2.1 \mathrm{~b}$ ) et $g$ est donné par le théorème de pureté en cohomologie étale, est commutatif : cela résulte tautologiquement de la construction des classes de cycles motiviques dans [Geisser et Levine 2001]. Par conséquent, ce diagramme s'étend en un diagramme commutatif de triangles exacts

$$
\begin{aligned}
& \mathbb{Z}(n-d)_{Y} \otimes \mathbb{Z}_{l}[-2 d] \stackrel{\mathrm{cl}_{Y}^{n-d}}{\longrightarrow} R \alpha_{*} \mathbb{Z}_{l}(n-d)_{Y}^{c}[-2 d] \rightarrow K(n-d)_{Y}[-2 d] \stackrel{+1}{\longrightarrow} \\
& f \downarrow \quad g \downarrow \quad h \downarrow \\
& R i_{\mathrm{Zar}}^{!} \mathbb{Z}(n)_{X} \otimes \mathbb{Z}_{l} \stackrel{\mathrm{cl}_{X}^{n}}{\longrightarrow} \quad R \alpha_{*} R i_{\mathrm{et}}^{!} \mathbb{Z}_{l}(n)_{X}^{c} \quad \rightarrow R i_{\mathrm{Zar}}^{!} K(n)_{X} \stackrel{+1}{\longrightarrow}
\end{aligned}
$$

(Rien n'est dit sur un choix privilégié de $h$.) Comme $f$ et $g$ sont des quasiisomorphismes, $h$ en est un aussi, d'où l'énoncé.

Comme $h$ est un morphisme dans la catégorie dérivée des faisceaux Zariski sur $Y$, la contravariance annoncée est tautologique pour $U \hookrightarrow X$ tel que $U \cap Y \neq \varnothing$, et elle est sans contenu lorsque $U \cap Y=\varnothing$. 
Lemme 3.10. Soit $l=p=$ car $k$. Soit $Y \subset X$ un couple lisse de codimension $d$. Alors il existe des homomorphismes

$$
H^{i-2 d}(Y, K(n-d)) \stackrel{h^{i}}{\longrightarrow} H_{Y}^{i}(X, K(n)) \quad(n \geq 0, i \in \mathbb{Z})
$$

contravariants pour les immersions ouvertes $U \hookrightarrow X$. Ce sont des isomorphismes pour $i \leq n+d$.

Démonstration. On raisonne comme dans la démonstration du lemme 3.9, en utilisant cette fois le théorème 2.6 b) et les résultats de Gros [1985]. D'après [Gros 1985, (3.5.3) et théorème 3.5.8], on a

$$
R^{q} i^{!} v_{r}(n)= \begin{cases}0 & \text { si } q \neq d, d+1 \\ v_{r}(n-d) & \text { si } q=d\end{cases}
$$

La formule (3-10) et sa compatibilité aux classes de cycles motiviques (elle est à la base de leur construction) fournit un diagramme commutatif dans $D\left(Y_{\mathrm{Zar}}\right)$

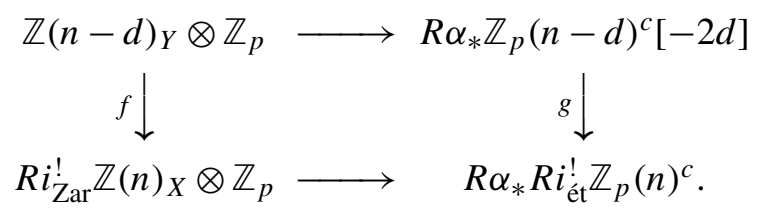

Il en résulte un morphisme

$$
K(n-d)_{Y} \stackrel{h}{\longrightarrow} R i_{\mathrm{Zar}}^{!} K(n)_{X}
$$

complétant le carré ci-dessus en un diagramme commutatif de triangles exacts. Ceci fournit les homomorphismes $h^{i}$ du lemme.

Dans le diagramme (3-11), $f$ est un isomorphisme (théorème 2.1 b)). Par (3-10), le cône de $g$ est acyclique en degrés $\leq n+d$. Par conséquent, le cône de $h$ est acyclique en degrés $\leq n+d$, ce qui donne la bijectivité de $h^{i}$ pour $i \leq n+d$.

3F. Fin de la démonstration du théorème 1.1. On prend maintenant $n=2$. Le résultat principal est :

Proposition 3.11. Pour $n=2$, l'homomorphisme $\alpha$ de (3-9) est surjectif de noyau $A^{1}\left(X, H^{2}(K(2))\right)$, uniquement divisible.

Démonstration. Notons $E_{2}^{a, b}=A^{a}\left(X, H^{b}(K(n))\right.$ : c'est la cohomologie d'un certain complexe de Cousin.

En utilisant les lemmes 3.9 et 3.10 , on trouve que $E_{1}^{a, b}=0$ pour

$l \neq p: a>2$ et $a+b<2 a ; a=2$ et $a+b \leq 4$.

$l=p: a>2$ et $a+b<2+a ; a=2$ et $a+b \leq 4$. 
(En particulier, $E_{2}^{2,2}=0$ puisque $K(0)=0$ !) On en déduit une suite exacte

$$
0 \rightarrow A^{1}\left(X, H^{2}(K(2))\right) \rightarrow H^{3}(X, K(2)) \rightarrow A^{0}\left(X, H^{3}(K(2))\right) \rightarrow 0 .
$$

Mais $A^{1}\left(X, H^{2}(K(2))\right)$ est l'homologie du complexe

$$
E_{1}^{0,2} \rightarrow E_{1}^{1,2} \rightarrow E_{1}^{2,2}
$$

dont tous les termes sont encore dans le domaine d'application des lemmes 3.9 et 3.10 (isomorphismes de pureté). D'après le lemme 3.7, ils sont uniquement divisibles, ainsi donc que $E_{2}^{1,2}$.

Le théorème 1.1 résulte maintenant de la proposition 3.6 , du lemme 3.7 , du lemme 3.8 et de la proposition 3.11.

3G. Un complément. Notons pour conclure :

Lemme 3.12. Le $\mathbb{Z}_{l}$-module $\left.H^{0}\left(X, \mathscr{H}_{\mathrm{cont}}^{3} \mathbb{Z}_{l}(2)\right)\right)$ est sans torsion.

Démonstration. On fait comme dans [Colliot-Thélène et Voisin 2010, théorème 3.1] (cet argument remonte à [Bloch et Srinivas 1983] pour la cohomologie de Betti) : le théorème de Merkurjev-Suslin implique que le faisceau $\mathscr{H}_{\text {cont }}^{3}\left(\mathbb{Z}_{l}(2)\right)$ est sans torsion. (Pour $l=p$, utiliser [Geisser et Levine 2000].)

\section{Cas d'un corps de base séparablement clos}

Soient $k$ un corps séparablement clos et $X$ une $k$-variété lisse. On veut préciser le théorème 1.1 dans ce cas, toujours dans l'esprit de [Colliot-Thélène et Voisin 2010].

4A. Lien avec les cycles de Tate. Le lemme suivant est démontré dans [ColliotThélène et Kahn 2011]. Il montre que les cycles de Tate entiers fournissent un bon analogue des cycles de Hodge entiers considérés dans [Colliot-Thélène et Voisin 2010] :

Lemme 4.1. Soient $G$ un groupe profini et $M$ un $\mathbb{Z}_{l}$-module de type fini muni d'une action continue de G. Soit

$$
M^{(1)}=\bigcup_{U} M^{U}
$$

où $U$ décrit les sous-groupes ouverts de G. Alors $M / M^{(1)}$ est sans torsion.

On en déduit :

Lemme 4.2. Supposons que $k$ soit la clôture séparable d'un corps de type fini et que $l \neq$ car $k$. Alors le groupe fini $C_{\text {tors }} d u$ théorème 1.1 est aussi le sous-groupe de torsion de

$$
\operatorname{Coker}\left(C H^{2}(X) \otimes \mathbb{Z}_{l} \rightarrow H_{\text {cont }}^{4}\left(X, \mathbb{Z}_{l}(2)\right)^{(1)}\right) .
$$


Sous la conjecture de Tate, ce conoyau est entièrement de torsion (pour X non nécessairement propre, $c f$. [Jannsen 1990, p. 114, Theorem 7.10 a)]).

On peut d'ailleurs supprimer l'hypothèse que $k$ soit la clôture séparable d'un corps de type fini. En général, écrivons $k=\bigcup_{\alpha} k_{\alpha}$, où $k_{\alpha}$ décrit l'ensemble (ordonné filtrant) des clôtures séparables des sous-corps de type fini de $k$ sur lesquels $X$ est définie. Pour tout $\alpha$, notons $X_{\alpha}$ le $k_{\alpha}$-modèle de $X$ correspondant. Si $k_{\alpha} \subset k_{\beta}$, on a des isomorphismes

$$
H_{\text {cont }}^{4}\left(X_{\alpha}, \mathbb{Z}_{l}(2)\right) \stackrel{\sim}{\longrightarrow} H_{\text {cont }}^{4}\left(X_{\beta}, \mathbb{Z}_{l}(2)\right) \stackrel{\sim}{\longrightarrow} H_{\text {cont }}^{4}\left(X, \mathbb{Z}_{l}(2)\right)
$$

et on peut définir

$$
H_{\text {cont }}^{4}\left(X, \mathbb{Z}_{l}(2)\right)^{(1)}:=\underset{\alpha}{\lim } H_{\text {cont }}^{4}\left(X_{\alpha}, \mathbb{Z}_{l}(2)\right)^{(1)} .
$$

Il s'agit en fait d'une limite inductive d'isomorphismes puisque, si $X$ est définie sur $k_{\alpha}^{0} \subset k_{\alpha}$ de type fini et de clôture séparable $k_{\alpha}$ et que $k_{\beta} \supset k_{\alpha}$, l'homomorphisme $\operatorname{Gal}\left(k_{\beta} / k_{\beta} k_{\alpha}^{0}\right) \rightarrow \operatorname{Gal}\left(k_{\alpha} / k_{\alpha}^{0}\right)$ est surjectif.

4B. Lien avec le groupe de Griffiths. Si $k=\mathbb{C}$ et $X$ est projective, Colliot-Thélène et Voisin ont établi un lien entre $H^{0}\left(X, \mathscr{H}^{3}(\mathbb{Q} / \mathbb{Z}(2))\right)$ et le groupe de Griffiths dans [Colliot-Thélène et Voisin 2010, §4.2]. Reprenons cette idée en l'amplifiant un peu.

Voici d'abord une définition de groupes de Griffiths et de groupes d'équivalence homologique dans le contexte $l$-adique. Supposons $k$ séparablement clos si $l \neq \operatorname{car} k$, et $k$ algébriquement clos si $l=$ car $k$. Par un argument bien connu [Bloch et Ogus 1974, Lemma 7.10; Bloch 2010, Lecture 1, Lemma 1.3]), pour toute $k$-variété lisse $X$, le sous-groupe de $C H^{n}(X)$ formé des cycles algébriquement équivalents à zéro est $l$-divisible; les diagrammes commutatifs

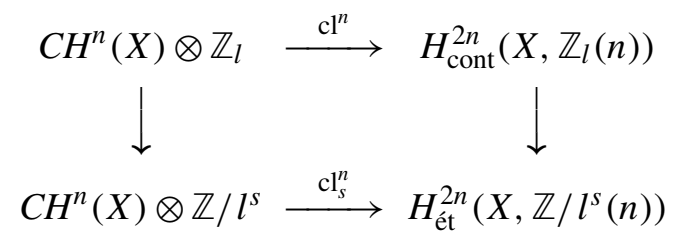

et l'isomorphisme

$$
H_{\text {cont }}^{2 n}\left(X, \mathbb{Z}_{l}(n)\right) \stackrel{\sim}{\longrightarrow} \lim _{s} H_{\text {êt }}^{2 n}\left(X, \mathbb{Z} / l^{s}(n)\right)
$$

montrent donc que $\mathrm{cl}_{s}^{n}$ et $\mathrm{cl}^{n}$ se factorisent à travers l'équivalence algébrique.

(Précisons. L'isomorphisme (4-1) est valable pourvu que le système projectif $\left(H_{\text {ét }}^{2 n-1}\left(X, \mathbb{Z} / l^{s}(n)\right)\right)_{s \geq 1}$ soit de Mittag-Leffler. Pour $l \neq$ car $k$ c'est vrai parce que les termes sont finis ; pour $l=\operatorname{car} k$ et $X$ projective c'est expliqué dans [ColliotThélène et al. 1983, p. 783], donc il faut a priori supposer $X$ projective dans ce cas.) 
Ceci donne un sens à :

Définition 4.3. Soit $X$ une $k$-variété lisse où $k$ est séparablement clos si $l \neq$ car $k$ et algébriquement clos si $l=\operatorname{car} k$; dans ce dernier cas, on suppose aussi $X$ projective. Soit $A \in\left\{\mathbb{Z}_{l}, \mathbb{Q}_{l}, \mathbb{Z} / l^{n}, \mathbb{Q}_{l} / \mathbb{Z}_{l}\right\}$. On note

$$
\begin{aligned}
\operatorname{Griff}^{n}(X, A) & =\operatorname{Ker}\left(A_{\mathrm{alg}}^{n}(X) \otimes A \stackrel{\mathrm{cl}^{n}}{\longrightarrow} H_{\mathrm{cont}}^{2 n}(X, A(n))\right) \\
A_{\mathrm{hom}}^{n}(X, A) & =\operatorname{Im}\left(A_{\mathrm{alg}}^{n}(X) \otimes A \stackrel{\mathrm{cl}^{n}}{\longrightarrow} H_{\mathrm{cont}}^{2 n}(X, A(n))\right) .
\end{aligned}
$$

Remarque 4.4. Si $k=\mathbb{C}$, on a $\operatorname{Griff}^{n}\left(X, \mathbb{Z}_{l}\right)=\operatorname{Griff}^{n}(X) \otimes \mathbb{Z}_{l}$ par l'isomorphisme de comparaison entre cohomologies de Betti et $l$-adique, où $\operatorname{Griff}^{n}(X)$ est défini à l'aide de la cohomologie de Betti.

On a la version $l$-adique de [Bloch et Ogus 1974, Theorem 7.3] :

Proposition 4.5. Supposons $l \neq \operatorname{car} k$. Notons $A_{\mathrm{alg}}^{n}(X)$ le groupe des cycles de codimension n sur X modulo l'équivalence algébrique. Dans la suite spectrale de coniveau

$$
E_{r}^{p, q} \Rightarrow H^{p+q}\left(X, \mathbb{Z}_{l}(n)\right)
$$

pour la cohomologie l-adique de X, on a un isomorphisme

$$
A_{\mathrm{alg}}^{n}(X) \otimes \mathbb{Z}_{l} \stackrel{\sim}{\longrightarrow} E_{2}^{n, n}
$$

induit par l'isomorphisme

$$
Z^{n}(X) \otimes \mathbb{Z}_{l} \stackrel{\sim}{\longrightarrow} E_{1}^{n, n}
$$

donné par les isomorphismes de pureté.

Démonstration. C'est la même que celle de [Bloch et Ogus 1974, preuve du théorème 7.3], mutatis mutandis. Plus précisément, la première étape est identique. Dans la deuxième étape, on remplace la désingularisation à la Hironaka des cycles de codimension $n$ de $X$ par une désingularisation à la de Jong [1996, Theorem 4.1]; pour obtenir des résultats entiers, on utilise le théorème de Ofer Gabber (travail en cours) disant qu'on peut trouver une telle désingularisation de degré premier à $l$. Enfin, l'argument transcendant de Bloch-Ogus pour prouver que équivalences algébrique et homologique coïncident pour les diviseurs sur une variété lisse $Y$ est remplacé par le suivant : par [Bloch et Ogus 1974, Lemma 7.10], le noyau de $C H^{n}(Y) \rightarrow A_{\text {alg }}^{n}(Y)$ est $l$-divisible, donc les suites exactes de Kummer

$$
\operatorname{Pic}(X) \stackrel{l^{n}}{\longrightarrow} \operatorname{Pic}(X) \rightarrow H^{2}\left(X, \mathbb{Z} / l^{n}(1)\right)
$$

définissent des injections

$$
0 \rightarrow A_{\mathrm{alg}}^{1}(X) / l^{n} \rightarrow H^{2}\left(X, \mathbb{Z} / l^{n}(1)\right)
$$


d'où à la limite

$$
0 \rightarrow A_{\text {alg }}^{1}(X) \otimes \mathbb{Z}_{l} \rightarrow H_{\text {cont }}^{2}\left(X, \mathbb{Z}_{l}(1)\right)
$$

puisque $A_{\text {alg }}^{1}(X)$ est un $\mathbb{Z}$-module de type fini.

Notons que ces arguments ne nécessitent pas que $X$ soit projective (pour le dernier, voir [Kahn 2006, théorème 3]). Si le théorème de Gabber n'évitait pas $l=p$, la démonstration s'étendrait à ce nombre premier.

Convention 4.6. À partir de maintenant, l est supposé différent de car $k$ sauf mention expresse du contraire. La raison essentielle pour cela est que cette restriction apparaît dans la proposition 4.5 (cf. le commentaire ci-dessus).

Corollaire 4.7 [Colliot-Thélène et Voisin 2010, Theorem 2.7]. On a une suite exacte

$$
H_{\text {cont }}^{3}\left(X, \mathbb{Z}_{l}(2)\right) \stackrel{\alpha}{\longrightarrow} H^{0}\left(X, \mathscr{H}_{\text {cont }}^{3}\left(\mathbb{Z}_{l}(2)\right)\right) \rightarrow \operatorname{Griff}^{2}\left(X, \mathbb{Z}_{l}\right) \rightarrow 0 .
$$

Démonstration. Cela résulte de la suite exacte

$$
H_{\text {cont }}^{3}\left(X, \mathbb{Z}_{l}(2)\right) \rightarrow E_{2}^{0,3} \rightarrow E_{2}^{2,2} \stackrel{c}{\longrightarrow} H_{\text {cont }}^{4}\left(X, \mathbb{Z}_{l}(2)\right)
$$

provenant de la suite spectrale de Bloch-Ogus en poids 2, de l'identification de $E_{2}^{0,3}$ à $H^{0}\left(X, \mathscr{H}_{\text {cont }}^{3}\left(\mathbb{Z}_{l}(2)\right)\right)$, de celle de $E_{2}^{2,2}$ à $A_{\text {alg }}^{2}(X) \otimes \mathbb{Z}_{l}$ (proposition 4.5) et de celle de $c$ à l'application classe de cycle.

L'analogue complexe du corollaire suivant devrait figurer dans [Colliot-Thélène et Voisin 2010] :

Corollaire 4.8. a) On a une suite exacte, modulo des groupes finis :

$$
H_{\text {cont }}^{3}\left(X, \mathbb{Z}_{l}(2)\right) \otimes \mathbb{Q} / \mathbb{Z} \rightarrow H^{0}\left(X, \mathscr{H}^{3}\left(\mathbb{Q}_{l} / \mathbb{Z}_{l}(2)\right)\right) \rightarrow \operatorname{Griff}^{2}\left(X, \mathbb{Z}_{l}\right) \otimes \mathbb{Q} / \mathbb{Z} \rightarrow 0
$$

(cf. définition 4.3).

b) Le groupe $H^{0}\left(X, \mathscr{H}^{3}\left(\mathbb{Q}_{l} / \mathbb{Z}_{l}(2)\right)\right)$ est dénombrable.

c) $S i$ car $k=0$, il existe $X / k$ projective lisse telle que son corang soit infini pour l convenable.

(Précisons : «modulo des groupes finis » signifie « dans la localisation de la catégorie des groupes abéliens relative à la sous-catégorie épaisse des groupes abéliens finis ».)

Démonstration. a) résulte du théorème 1.1 et du corollaire 4.7. Pour b), le terme de gauche dans la suite de a) est de cotype fini, donc dénombrable, et le terme de droite l'est aussi (propriété classique des cycles modulo l'équivalence algébrique). Enfin, d'après [Schoen 2002], on a des exemples de $X$ et de nombres premiers $l$ (même sur $\overline{\mathbb{Q}}$ ) où $\operatorname{Griff}^{2}(X) / l$ est infini ; en utilisant [Colliot-Thélène et Voisin 
2010, Proposition 4.1] (voir aussi corollaire 4.15 du présent article), on en déduit que $\operatorname{Griff}^{2}(X) \otimes \mathbb{Q}_{l} / \mathbb{Z}_{l}$ est de corang infini.

4C. Quelques calculs de groupes de torsion. On veut maintenant préciser le corollaire 4.8 a) en décrivant explicitement le noyau de l'application

$$
H_{\text {cont }}^{3}\left(X, \mathbb{Z}_{l}(2)\right) \otimes \mathbb{Q} / \mathbb{Z} \rightarrow H^{0}\left(X, \mathscr{H}_{\text {cont }}^{3}\left(\mathbb{Z}_{l}(2)\right)\right) \otimes \mathbb{Q} / \mathbb{Z} .
$$

Définition 4.9. Soit $A$ un $\mathbb{Z}_{l}$-module de la forme $\mathbb{Z}_{l}, \mathbb{Q}_{l}, \mathbb{Z} / l^{n}, \mathbb{Q}_{l} / \mathbb{Z}_{l}$. On note $N H_{\text {cont }}^{3}(X, A)$ le premier cran de la filtration par le coniveau sur $H_{\text {cont }}^{3}(X, A)$ et

$$
H_{\mathrm{tr}}^{3}(X, A)=\frac{H_{\mathrm{cont}}^{3}(X, A)}{N H_{\mathrm{cont}}^{3}(X, A)} .
$$

Si on a un twist à la Tate, on note $N H_{\text {cont }}^{3}(X, A(n)):=N H_{\text {cont }}^{3}(X, A)(n)$.

Notons que $N H_{\text {cont }}^{3}(X, A(2))=H^{3}(X, A(2))$ (cohomologie motivique de Nisnevich) pour $A=\mathbb{Z} / l^{n}$ ou $\mathbb{Q}_{l} / \mathbb{Z}_{l}$, d'après la proposition 2.11 .

Remarque 4.10. Si $X$ est propre, les $\mathbb{Z}_{l}$-modules $H_{\mathrm{tr}}^{3}(X, A)$ sont des invariants birationnels de $X$, avec l'action de $\operatorname{Gal}\left(k / k_{0}\right)$ si $X$ est défini sur un sous-corps $k_{0}$ de clôture séparable $k$. C'est dû à Grothendieck [1968, 9.4].

Le lemme 3.12 implique :

Lemme 4.11. Le $\mathbb{Z}_{l}$-module de type fini $H_{\mathrm{tr}}^{3}\left(X, \mathbb{Z}_{l}(2)\right)$ est sans torsion.

Par définition de $N H_{\text {cont }}^{3}\left(X, \mathbb{Z}_{l}(2)\right)$, la suite exacte du corollaire 4.7 se raffine en une suite exacte :

$$
0 \rightarrow H_{\mathrm{tr}}^{3}\left(X, \mathbb{Z}_{l}(2)\right) \rightarrow H^{0}\left(X, \mathscr{H}_{\mathrm{cont}}^{3}\left(\mathbb{Z}_{l}(2)\right)\right) \rightarrow \operatorname{Griff}^{2}\left(X, \mathbb{Z}_{l}\right) \rightarrow 0
$$

qui montre incidemment que $\operatorname{Griff}^{2}\left(X, \mathbb{Z}_{l}\right)$ est un invariant birationnel pour $X$ propre et lisse (cf. remarque 4.10). En réutilisant le lemme 3.12, on en déduit :

Proposition 4.12. On a une suite exacte

$$
\begin{aligned}
0 \rightarrow \operatorname{Griff}^{2}\left(X, \mathbb{Z}_{l}\right)_{\text {tors }} & \rightarrow H_{\text {tr }}^{3}\left(X, \mathbb{Z}_{l}(2)\right) \otimes \mathbb{Q} / \mathbb{Z} \\
& \rightarrow H^{0}\left(X, \mathscr{H}_{\text {cont }}^{3}\left(\mathbb{Z}_{l}(2)\right)\right) \otimes \mathbb{Q} / \mathbb{Z} \rightarrow \operatorname{Griff}^{2}\left(X, \mathbb{Z}_{l}\right) \otimes \mathbb{Q} / \mathbb{Z} \rightarrow 0 . \square
\end{aligned}
$$

Remarque 4.13. Dans cette remarque, nous adoptons la convention contravariante pour les motifs purs sur un corps. Supposons que $X$, de dimension $d$, vérifie la conjecture standard $\mathrm{C}$ et la conjecture de nilpotence suivante : l'idéal

$$
\operatorname{Ker}\left(C H^{d}(X \times X) \otimes \mathbb{Q} \rightarrow A_{\text {num }}^{d}(X \times X) \otimes \mathbb{Q}\right)
$$

de l'anneau des correspondances de Chow est nilpotent. Ces propriétés sont vérifiées par exemple si $X$ est une variété abélienne : voir [Kleiman 1968] pour la première (résultat de Lieberman et Kleiman) et [Kimura 2005] pour la seconde. Alors le 
motif numérique de $X$ admet une décomposition de Künneth, qui se relève en une décomposition de Chow-Künneth de son motif de Chow :

$$
h(X)=\bigoplus_{i=0}^{2 d} h^{i}(X)
$$

Mais le théorème de semi-simplicité de Jannsen [1992] implique que chaque facteur numérique $h_{\text {num }}^{i}(X)$ admet une décomposition plus fine, provenant de sa décomposition isotypique :

$$
h_{\text {num }}^{i}(X)=\bigoplus_{j=0}^{i / 2} h_{\text {num }}^{i, j}(X)(-j)
$$

où $h_{\text {num }}^{i, j}(X)$ est effectif mais aucun facteur simple de $h_{\text {num }}^{i, j}(X)(1)$ n'est effectif. Cette décomposition se relève de nouveau pour donner une décomposition de Chow-Künneth raffinée (voir [Kahn et al. 2007, théorème 7.7.3]) :

$$
h(X)=\bigoplus_{i=0}^{2 d} \bigoplus_{j=0}^{i / 2} h^{i, j}(X)(-j) .
$$

Notons Ab la catégorie des groupes abéliens, $A$ le quotient de Ab par la souscatégorie épaisse des groupes abéliens d'exposant fini et, pour tout anneau commutatif $R$, Chow $(k, R)$ la catégorie des motifs de Chow à coefficients dans $R$. On observe que le foncteur

$$
\operatorname{Hom}(-,-): \operatorname{Chow}(k, \mathbb{Z})^{\mathrm{op}} \times \operatorname{Chow}(k, \mathbb{Z}) \rightarrow \mathrm{Ab}
$$

s'étend en un foncteur

$$
\operatorname{Hom}(-,-): \operatorname{Chow}(k, \mathbb{Q})^{\text {op }} \times \operatorname{Chow}(k, \mathbb{Q}) \rightarrow \mathscr{A} \text {. }
$$

Par construction, $H_{\mathrm{tr}}^{3}\left(X, \mathbb{Q}_{l}\right)=H_{\text {cont }}^{*}\left(h^{3,0}(X), \mathbb{Q}_{l}\right)$. Au moins si $d=3$, on peut montrer que, d'autre part,

$$
\operatorname{Griff}^{2}\left(X, \mathbb{Z}_{l}\right) \simeq \operatorname{Hom}\left(h^{3,0}(X), \mathbb{L}\right) \otimes \mathbb{Z}_{l}
$$

où $\mathbb{L}$ est le motif de Lefschetz et l'isomorphisme est dans $\mathcal{A}$. Ainsi, la proposition 4.12 et le théorème 1.1 montrent que (si $d=3$ ) la nullité de $h^{3,0}(X)$ entraîne la finitude de $H^{0}\left(X, \mathscr{H}^{3}\left(\mathbb{Q}_{l} / \mathbb{Z}_{l}(2)\right)\right)$.

D'autre part, la conjecture de Bloch-Beilinson-Murre [Jannsen 1994] implique que la nullité de $h^{3,0}(X)$ découle de celle de $H_{\mathrm{tr}}^{3}\left(X, \mathbb{Q}_{l}\right)$ : conjecturalement, celle-ci est donc suffisante pour impliquer la finitude du groupe $H^{0}\left(X, \mathscr{H}^{3}\left(\mathbb{Q}_{l} / \mathbb{Z}_{l}(2)\right)\right.$ ) (au moins si $\operatorname{dim} X=3$ ). 
Ceci est une variante de la conjecture 4.5 de [Colliot-Thélène et Voisin 2010]. On verra au théorème $5.2 \mathrm{c}$ ) qu'elle est vraie si $k$ est la clôture algébrique d'un corps fini $k_{0}$ et que $X$ provient de la classe $B_{\text {Tate }}\left(k_{0}\right)$ de [Kahn 2003].

On peut se demander si la réciproque est vraie. Elle est fausse (théorème 5.6).

Le lemme 4.11 et la proposition 2.11 donnent un diagramme commutatif de suites exactes

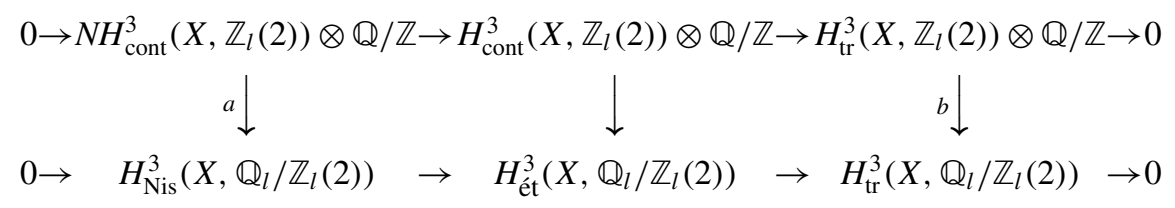

dans lequel la flèche verticale centrale est injective de conoyau fini, isomorphe à $H_{\text {cont }}^{4}\left(X, \mathbb{Z}_{l}(2)\right)_{\text {tors. }}$. Par le lemme du serpent, on en déduit :

Proposition 4.14. Avec les notations de (4-2), a est injective et on a une suite exacte

$$
0 \rightarrow \operatorname{Ker} b \rightarrow \text { Coker } a \rightarrow H_{\text {cont }}^{4}\left(X, \mathbb{Z}_{l}(2)\right)_{\text {tors }} \rightarrow \text { Coker } b \rightarrow 0 .
$$

Voici une application de la proposition 4.14.

Corollaire 4.15. Soit $H^{3}\left(X, \mathbb{Q}_{l} / \mathbb{Z}_{l}(2)\right)^{0}$ le noyau de la composition

$$
H^{3}\left(X, \mathbb{Q}_{l} / \mathbb{Z}_{l}(2)\right) \rightarrow H_{\text {êt }}^{3}\left(X, \mathbb{Q}_{l} / \mathbb{Z}_{l}(2)\right) \rightarrow H_{\text {cont }}^{4}\left(X, \mathbb{Z}_{l}(2)\right)_{\text {tors }}
$$

Alors $\operatorname{Im} a \subset H^{3}\left(X, \mathbb{Q}_{l} / \mathbb{Z}_{l}(2)\right)^{0}$ (notations de (4-2)) et on a un isomorphisme

$$
\operatorname{Griff}^{2}\left(X, \mathbb{Z}_{l}\right)_{\text {tors }} \stackrel{\sim}{\longrightarrow} \operatorname{Ker} b \stackrel{\sim}{\longrightarrow} \text { Coker } a^{0}
$$

où $a^{0}: N H_{\text {cont }}^{3}\left(X, \mathbb{Z}_{l}(2)\right) \otimes \mathbb{Q} / \mathbb{Z} \stackrel{a}{\longrightarrow} H^{3}\left(X, \mathbb{Q}_{l} / \mathbb{Z}_{l}(2)\right)^{0}$ est l'application induite para.

Démonstration. La première assertion est évidente. Notons $a^{0}$ l'application induite : la suite exacte de la proposition 4.14 induit donc un isomorphisme

$$
\operatorname{Ker} b \stackrel{\sim}{\longrightarrow} \operatorname{Coker} a^{0} .
$$

D'autre part, la suite exacte de la proposition 4.12 s'insère dans un diagramme commutatif de suites exactes

$0 \rightarrow \operatorname{Griff}^{2}\left(X, \mathbb{Z}_{l}\right)_{\mathrm{tors}} \rightarrow H_{\mathrm{tr}}^{3}\left(X, \mathbb{Z}_{l}(2)\right) \otimes \mathbb{Q} / \mathbb{Z} \rightarrow H^{0}\left(X, \mathscr{H}_{\mathrm{cont}}^{3}\left(\mathbb{Z}_{l}(2)\right)\right) \otimes \mathbb{Q} / \mathbb{Z}$

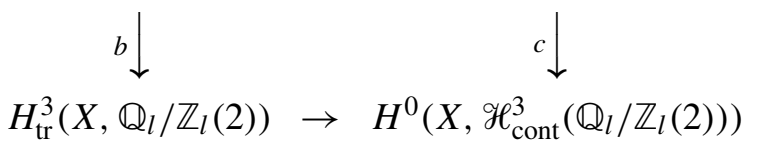

Comme le faisceau $\mathscr{H}_{\text {cont }}^{3}\left(\mathbb{Z}_{l}(2)\right)$ est sans torsion, la suite $0 \rightarrow H^{0}\left(X, \mathscr{H}_{\text {cont }}^{3}\left(\mathbb{Z}_{l}(2)\right)\right) \rightarrow H^{0}\left(X, \mathscr{H}_{\text {cont }}^{3}\left(\mathbb{Q}_{l}(2)\right)\right) \rightarrow H^{0}\left(X, \mathscr{H}_{\text {cont }}^{3}\left(\mathbb{Q}_{l} / \mathbb{Z}_{l}(2)\right)\right)$ 
est exacte, ce qui signifie que $c$ est injective dans le diagramme ci-dessus. On en déduit un isomorphisme

$$
\operatorname{Griff}^{2}\left(X, \mathbb{Z}_{l}\right)_{\text {tors }} \stackrel{\sim}{\longrightarrow} \operatorname{Ker} b
$$

d'où le corollaire.

4D. Les homomorphismes de groupes $A_{\mathrm{hom}}^{2}\left(X, \mathbb{Z}_{l}\right) \otimes \mathbb{Q}_{l} / \mathbb{Z}_{l} \rightarrow A_{\mathrm{hom}}^{2}\left(X, \mathbb{Q}_{l} / \mathbb{Z}_{l}\right)$ et $\operatorname{Griff}^{2}\left(X, \mathbb{Z}_{l}\right) \otimes \mathbb{Q}_{l} / \mathbb{Z}_{l} \rightarrow \operatorname{Griff}^{2}\left(X, \mathbb{Q}_{l} / \mathbb{Z}_{l}\right)$. On garde les notations de la définition 4.3 .

Proposition 4.16. On a des suites exactes

$$
\begin{aligned}
A_{\text {hom }}^{2}\left(X, \mathbb{Z}_{l}\right)_{\text {tors }} \rightarrow \operatorname{Griff}^{2}\left(X, \mathbb{Z}_{l}\right) & \otimes \mathbb{Q}_{l} / \mathbb{Z}_{l} \rightarrow \operatorname{Griff}^{2}\left(X, \mathbb{Q}_{l} / \mathbb{Z}_{l}\right) \\
& \rightarrow A_{\text {hom }}^{2}\left(X, \mathbb{Z}_{l}\right) \otimes \mathbb{Q}_{l} / \mathbb{Z}_{l} \rightarrow A_{\text {hom }}^{2}\left(X, \mathbb{Q}_{l} / \mathbb{Z}_{l}\right) \rightarrow 0
\end{aligned}
$$

et

$$
C_{\text {tors }} \rightarrow A_{\text {hom }}^{2}\left(X, \mathbb{Z}_{l}\right) \otimes \mathbb{Q}_{l} / \mathbb{Z}_{l} \rightarrow A_{\text {hom }}^{2}\left(X, \mathbb{Q}_{l} / \mathbb{Z}_{l}\right)
$$

où C est comme dans (1-1) (voir théorème 1.1). En particulier, l'application

$$
\operatorname{Griff}^{2}\left(X, \mathbb{Z}_{l}\right) \otimes \mathbb{Q}_{l} / \mathbb{Z}_{l} \rightarrow \operatorname{Griff}^{2}\left(X, \mathbb{Q}_{l} / \mathbb{Z}_{l}\right)
$$

est de noyau et de conoyau finis.

Démonstration. On a un diagramme commutatif de suites exactes

$$
\begin{gathered}
A_{\text {hom }}^{2}\left(X, \mathbb{Z}_{l}\right)_{\text {tors }} \rightarrow \operatorname{Griff}^{2}\left(X, \mathbb{Z}_{l}\right) \otimes \mathbb{Q} / \mathbb{Z} \rightarrow A_{\text {alg }}^{2}\left(X, \mathbb{Z}_{l}\right) \otimes \mathbb{Q} / \mathbb{Z} \rightarrow A_{\text {hom }}^{2}\left(X, \mathbb{Z}_{l}\right) \otimes \mathbb{Q} / \mathbb{Z} \rightarrow 0 \\
\qquad \rightarrow \operatorname{Griff}^{2}\left(X, \mathbb{Q}_{l} / \mathbb{Z}_{l}\right) \rightarrow A_{\text {alg }}^{2}\left(X, \mathbb{Q}_{l} / \mathbb{Z}_{l}\right) \rightarrow A_{\text {hom }}^{2}\left(X, \mathbb{Q}_{l} / \mathbb{Z}_{l}\right) \rightarrow 0
\end{gathered}
$$

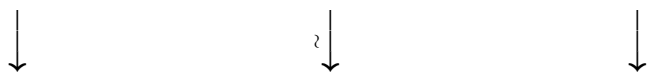

qui donne la première suite de la proposition, par application du lemme du serpent. Pour la seconde, on utilise le diagramme commutatif de suites exactes

$$
\begin{gathered}
\left(\frac{H_{\text {cont }}^{4}\left(X, \mathbb{Z}_{l}(2)\right)}{N^{2} H_{\text {cont }}^{4}\left(X, \mathbb{Z}_{l}(2)\right)}\right)_{\text {tors }} \rightarrow A_{\text {hom }}^{2}\left(X, \mathbb{Z}_{l}\right) \otimes \mathbb{Q} / \mathbb{Z} \rightarrow H_{\text {cont }}^{4}\left(X, \mathbb{Z}_{l}\right) \otimes \mathbb{Q} / \mathbb{Z} \\
0 \quad \rightarrow \quad A_{\text {hom }}^{2}\left(X, \mathbb{Q}_{l} / \mathbb{Z}_{l}\right) \rightarrow H_{\text {ét }}^{4}\left(X, \mathbb{Q}_{l} / \mathbb{Z}_{l}(2)\right)
\end{gathered}
$$

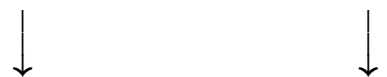

en remarquant que la flèche verticale de droite est injective.

4E. Le sous-groupe de torsion de $\boldsymbol{C H}^{2}\left(X, \mathbb{Z}_{l}\right)_{\text {alg. }}$ Terminons cette analyse de la torsion en déterminant celle de $C H^{2}\left(X, \mathbb{Z}_{l}\right)_{\text {alg }}$, sous-groupe de $C H^{2}(X) \otimes \mathbb{Z}_{l}$ formé des classes de cycles algébriquement équivalentes à zéro, lorsque $X$ est propre : voir corollaire 4.21. Pour cela nous avons besoin de la proposition suivante : 
Proposition 4.17. Supposons $k$ séparablement clos, $X / k$ propre et lisse et $i<2 n$. Soit $l$ un nombre premier; si $l=\mathrm{car} k$, on suppose $k$ algébriquement clos. Alors l'image de l'application cycle (3-1) est égale à $H_{\mathrm{cont}}^{i}\left(X, \mathbb{Z}_{l}(n)\right)_{\mathrm{tors}}$. En particulier, $H_{\mathrm{et}}^{i}(X, \mathbb{Z}(n))$ est extension d'un groupe de torsion (fini pour $l \neq p$ ) par un groupe divisible, et

$$
H_{\text {ét }}^{i}(X, \mathbb{Z}(n)) \otimes \mathbb{Q}_{l} / \mathbb{Z}_{l}=0 .
$$

Démonstration. Étant donné le corollaire 3.5, il suffit de montrer que (3-1) a une image de torsion. On reprend les arguments de Colliot-Thélène et Raskind [1985] : d'après le théorème $2.1 \mathrm{a}$ ) et le $\S 2 \mathrm{C}$, on a

$$
H_{\mathrm{et}}^{i}(X, \mathbb{Z}(n))=\underset{\alpha}{\lim } H_{\mathrm{et}}^{i}\left(X_{\alpha}, \mathbb{Z}(n)\right)
$$

où $X_{\alpha}$ parcourt un ensemble ordonné filtrant de modèles de $X$ sur des sous-corps $F_{\alpha}$ de type fini sur le corps premier. Il suffit donc de savoir que $H^{i}\left(X, \mathbb{Z}_{l}(n)\right)^{G}$ est de torsion, où $G$ est le groupe de Galois absolu de $F_{\alpha}^{p^{-\infty}}$. On le voit en se ramenant au cas d'un corps de base fini par changement de base propre et lisse (SGA4 pour $l \neq p$, [Gros et Suwa 1988a, p. 590, théorème 2.1] pour $l=p$ ), où cela résulte de la démonstration par Deligne de la conjecture de Weil [Deligne 1974] pour $l \neq p$ et du complément de Katz et Messing [1974] pour $l=p$.

Remarque 4.18. Supposons $n=2$. En utilisant la suite spectrale de coniveau de la remarque 2.4 , on obtient un isomorphisme $H^{i}(X, \mathbb{Z}(2)) \simeq H^{i-2}\left(X, \mathscr{K}_{2}\right)$ : alors l'énoncé n'est autre que celui de [Colliot-Thélène et Raskind 1985, Theorem 1.8 et 2.2] pour $l \neq p$, et de [Gros et Suwa 1988a, p. 604, corollaire 2.2 et p. 605, théorème 3.1] pour $l=p$.

Corollaire 4.19. Sous ces hypothèses, les homomorphismes

$$
\begin{aligned}
& H^{3}\left(X, \mathbb{Q}_{l} / \mathbb{Z}_{l}(2)\right) \rightarrow C H^{2}(X)\{l\}, \\
& H_{\text {èt }}^{3}\left(X, \mathbb{Q}_{l} / \mathbb{Z}_{l}(2)\right) \rightarrow H_{\text {êt }}^{4}(X, \mathbb{Z}(2))\{l\}
\end{aligned}
$$

sont bijectifs.

Démonstration. Pour le second, cela résulte de la suite exacte des coefficients universels et de la proposition 4.17 appliquée pour $(i, n)=(3,2)$. Pour le premier, même raisonnement en utilisant le fait que l'homomorphisme

$$
H^{i}(X, \mathbb{Z}(2)) \rightarrow H_{\mathrm{et}}^{i}(X, \mathbb{Z}(2))
$$

est bijectif pour $i \leq 3$ par (2-6) (qui résulte en poids 2 du théorème de MerkurjevSuslin).

En particulier, on obtient une injection

$$
H_{\text {cont }}^{3}\left(X, \mathbb{Z}_{l}(2)\right) \otimes \mathbb{Q}_{l} / \mathbb{Z}_{l} \hookrightarrow H_{\text {ét }}^{4}(X, \mathbb{Z}(2)) .
$$


Corollaire 4.20. Sous les mêmes hypothèses, soit $N$ le noyau de l'homomorphisme $H_{\mathrm{et}}^{4}(X, \mathbb{Z}(2)) \otimes \mathbb{Z}_{l} \rightarrow H_{\text {cont }}^{4}\left(X, \mathbb{Z}_{l}(2)\right)$. Alors (4-3) induit un isomorphisme

$$
H_{\text {cont }}^{3}\left(X, \mathbb{Z}_{l}(2)\right) \otimes \mathbb{Q}_{l} / \mathbb{Z}_{l} \stackrel{\sim}{\rightarrow} N_{\text {tors }} .
$$

Corollaire 4.21. Sous les mêmes hypothèses, on a un isomorphisme canonique:

$$
C H^{2}\left(X, \mathbb{Z}_{l}\right)_{\text {alg }}\{l\} \stackrel{\sim}{\rightarrow} N H_{\text {cont }}^{3}\left(X, \mathbb{Z}_{l}(2)\right) \otimes \mathbb{Q} / \mathbb{Z} .
$$

Démonstration. On va réutiliser le complexe $K(2)$ de la définition 3.2. Considérons le diagramme commutatif de suites exactes :

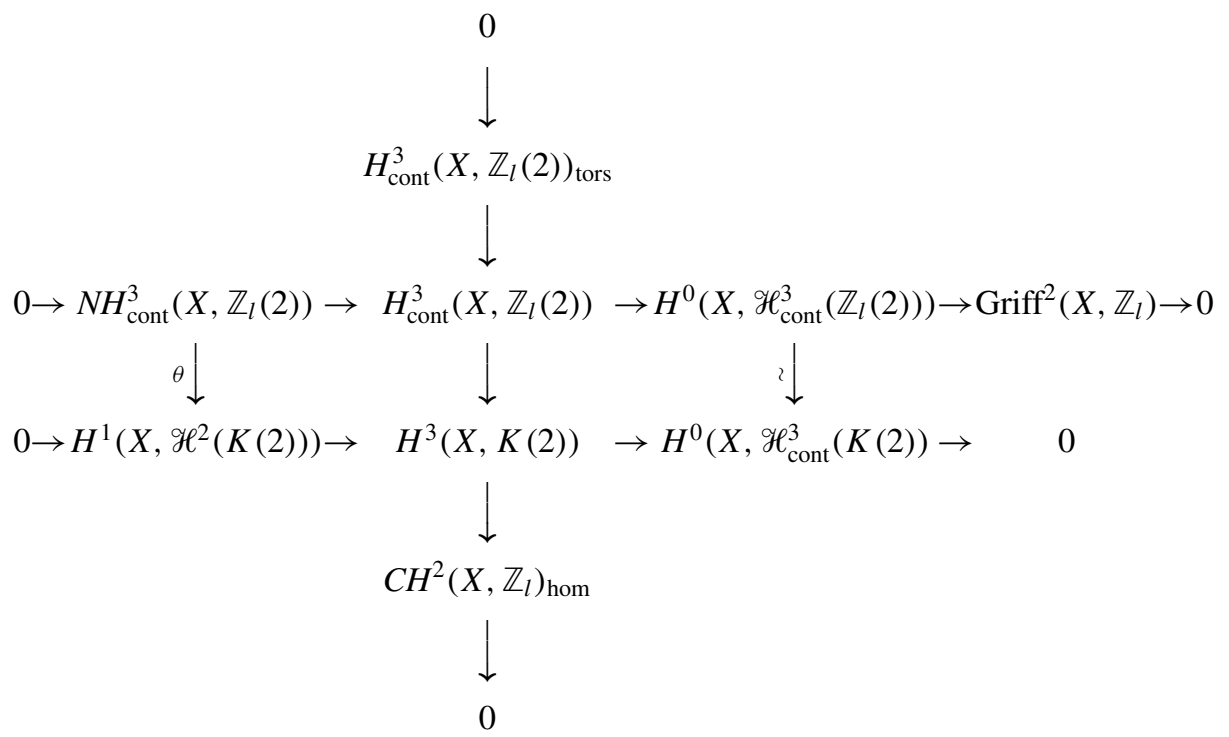

où $C H^{2}\left(X, \mathbb{Z}_{l}\right)_{\text {hom }}$ est le noyau de la classe de cycle sur $C H^{2}(X) \otimes \mathbb{Z}_{l}$. L'exactitude à gauche de la suite verticale découle de la proposition 4.17 , celle de la première suite horizontale du corollaire 4.7, la seconde suite horizontale est (3-12), enfin l'isomorphisme vertical est le lemme 3.8. La flèche $\theta$ est induite par le diagramme.

Tout d'abord, le lemme 3.12 implique via ce diagramme que

$$
N H_{\text {cont }}^{3}\left(X, \mathbb{Z}_{l}(2)\right)_{\text {tors }} \stackrel{\sim}{\longrightarrow} H_{\text {cont }}^{3}\left(X, \mathbb{Z}_{l}(2)\right)_{\text {tors }} .
$$

Appliquons maintenant le lemme du serpent aux deux suites exactes horizontales : on obtient un isomorphisme

$$
\operatorname{Ker} \theta \stackrel{\sim}{\rightarrow} H_{\text {cont }}^{3}\left(X, \mathbb{Z}_{l}(2)\right)_{\text {tors }}
$$

et une suite exacte

$$
0 \rightarrow \operatorname{Coker} \theta \rightarrow C H^{2}\left(X, \mathbb{Z}_{l}\right)_{\text {hom }} \stackrel{\phi}{\longrightarrow} \operatorname{Griff}^{2}\left(X, \mathbb{Z}_{l}\right) \rightarrow 0
$$


et on calcule que $\phi$ est la projection naturelle. Finalement on obtient une suite exacte

$$
0 \rightarrow N H_{\text {cont }}^{3}\left(X, \mathbb{Z}_{l}(2)\right) / \text { tors } \rightarrow H^{1}\left(X, \mathscr{H}^{2}(K(2))\right) \rightarrow C H^{2}\left(X, \mathbb{Z}_{l}\right)_{\mathrm{alg}} \rightarrow 0
$$

et l'isomorphisme du corollaire découle maintenant de la proposition 3.11 et de la suite exacte des Tor à coefficients $\mathbb{Q} / \mathbb{Z}$.

Remarque 4.22. Si $k$ est la clôture algébrique d'un corps fini, alors $C H^{n}\left(X, \mathbb{Z}_{l}\right)_{\text {alg }}$ est un groupe de torsion pour toute $k$-variété projective lisse $X$ et tout $n \geq 0$ (réduction au cas d'une courbe par l'argument de correspondances de Bloch, cf. [Schoen 1995, preuve de la proposition 2.7]). En particulier, le corollaire 4.21 décrit le groupe $C H^{2}\left(X, \mathbb{Z}_{l}\right)_{\text {alg }}$ tout entier. (Voir aussi §5C.)

\section{Cas d'un corps de base fini et de sa clôture algébrique}

5A. Cas d'un corps fini. Soit $k$ un corps fini. Rappelons d'abord la classe $B_{\text {Tate }}(k)$ de [Kahn 2003, définition 1 b)]

Définition 5.1. Une $k$-variété projective lisse $X$ est dans $B_{\text {Tate }}(k)$ si

(i) Il existe une $k$-variété abélienne $A$ et une extension finie $k^{\prime} / k$ telles que le motif de Chow de $X_{k^{\prime}}$ à coefficients rationnels soit facteur direct de celui de $A_{k^{\prime}}$.

(ii) $X$ vérifie la conjecture de Tate (sur l'ordre des pôles de $\zeta(X, s)$ aux entiers $\geq 0$ ).

On sait montrer qu'étant donné (i), (ii) est conséquence de (donc équivalent à) la conjecture de Tate cohomologique pour la cohomologie $l$-adique, pour un nombre premier $l$ donné pouvant être égal à la caractéristique de $k$ (cela résulte de [Kahn 2003, lemme 1.9], cf. [Colliot-Thélène et Kahn 2011, remarque 3.10]).

Considérons les notations de la preuve de la proposition 3.6. Si $k$ est fini et si $X \in B_{\text {Tate }}(k), K_{\text {ét }}$ et $C_{\text {ét }}$ sont finis (ibid., théorème 3.6 et lemme 3.7), donc $K=K_{\text {ét }}=C_{\text {ét }}=0$ et (3-5) devient un isomorphisme

$$
H^{0}\left(X, \mathscr{H}_{\text {ét }}^{3}\left(\mathbb{Q}_{l} / \mathbb{Z}_{l}(2)\right)\right) \stackrel{\sim}{\longrightarrow} C .
$$

En particulier, $H^{0}\left(X, \mathscr{H}_{\mathrm{ett}}^{3}\left(\mathbb{Q}_{l} / \mathbb{Z}_{l}(2)\right)\right)$ est fini et $H^{0}\left(X, \mathscr{H}_{\text {cont }}^{3}\left(\mathbb{Z}_{l}(2)\right)\right) \otimes \mathbb{Q} / \mathbb{Z}=0$ (théorème 1.1). En réalité, même le groupe $H^{0}\left(X, \mathscr{H}_{\mathrm{ett}}^{3}(\mathbb{Q} / \mathbb{Z}(2))\right)$ est fini : cela résulte de la proposition 2.9 et de la génération finie de $H_{\text {ét }}^{4}(X, \mathbb{Z}(2))$ [Kahn 2003, corollaire $3.8 \mathrm{c}$ ) et e)].

Conjecturalement, toute variété projective lisse est dans $B_{\text {Tate }}(k)$. 
5B. Cas de la clôture algébrique d'un corps fini. Le but de ce numéro est de démontrer :

Théorème 5.2. Soient $k$ la clôture algébrique d'un corps fini $k_{0}, X_{0} \in B_{\text {Tate }}\left(k_{0}\right)$, $X=X_{0} \otimes_{k_{0}} k$ et $l \neq$ car $k$.

a) $\operatorname{Griff}^{2}\left(X, \mathbb{Z}_{l}\right)$ est de torsion.

b) On a une suite exacte

$$
0 \rightarrow \operatorname{Griff}^{2}\left(X, \mathbb{Z}_{l}\right) \rightarrow H_{\text {tr }}^{3}\left(X, \mathbb{Z}_{l}(2)\right) \otimes \mathbb{Q} / \mathbb{Z} \rightarrow H^{0}\left(X, \mathscr{H}_{\text {cont }}^{3}\left(\mathbb{Z}_{l}(2)\right)\right) \otimes \mathbb{Q} / \mathbb{Z} \rightarrow 0 .
$$

c) $\operatorname{Si} H_{\mathrm{tr}}^{3}\left(X, \mathbb{Q}_{l}(2)\right)=0$, le groupe $H^{0}\left(X, \mathscr{H}^{3}\left(\mathbb{Q}_{l} / \mathbb{Z}_{l}(2)\right)\right)$ est fini; dans ce cas, il est isomorphe à $C_{\text {tors. }}$.

Remarque 5.3. Le corollaire 4.15 donne une autre description de $\operatorname{Griff}^{2}\left(X, \mathbb{Z}_{l}\right)$.

Démonstration. a) $\Rightarrow$ b) par la proposition 4.12 et b) $\Rightarrow$ c) par le théorème 1.1. Montrons a). Soit $k_{1}$ une extension finie de $k_{0}$, et $X_{1}=X_{0} \otimes_{k_{0}} k_{1}$. D'après [Kahn 2003, théorème 3.6 et corollaire 3.8 e)], l'homomorphisme

$$
H_{\text {ét }}^{4}\left(X_{1}, \mathbb{Z}(2)\right) \otimes \mathbb{Z}_{l} \rightarrow H_{\text {cont }}^{4}\left(X_{1}, \mathbb{Z}_{l}(2)\right)
$$

est bijectif. D'après (2-7), l'homomorphisme

$$
C H^{2}\left(X_{1}\right) \otimes \mathbb{Z}_{l} \rightarrow H_{\text {cont }}^{4}\left(X_{1}, \mathbb{Z}_{l}(2)\right)
$$

est donc injectif. Or dans la suite exacte

$0 \rightarrow H^{1}\left(G_{1}, H_{\text {cont }}^{3}\left(X, \mathbb{Z}_{l}(2)\right)\right) \rightarrow H_{\text {cont }}^{4}\left(X_{1}, \mathbb{Z}_{l}(2)\right) \rightarrow H_{\text {cont }}^{4}\left(X, \mathbb{Z}_{l}(2)\right)^{G_{1}} \rightarrow 0$

(où $G_{1}=\operatorname{Gal}\left(k / k_{1}\right)$ ), le groupe de gauche est fini d'après Weil I [Deligne 1974]. Il en résulte que le noyau de

$$
C H^{2}\left(X_{1}\right) \otimes \mathbb{Z}_{l} \rightarrow H_{\text {cont }}^{4}\left(X, \mathbb{Z}_{l}(2)\right)
$$

est fini pour tout $k_{1}$, d'où la conclusion en passant à la limite.

5C. Un exemple de Schoen. J'avais initialement pensé que la réciproque du théorème $5.2 \mathrm{c}$ ) est vraie. En réalité elle est fausse : cela résulte d'un calcul de $\mathrm{C}$. Schoen [1995]. Dans cet article, Schoen considère $X=E^{3}$ sur $k=\overline{\mathbb{F}}_{p}$, où $E$ est la courbe elliptique d'équation $x^{3}+y^{3}+z^{3}=0$, et montre que, si $p \equiv 1(\bmod 3)$ :

$$
\operatorname{Griff}^{2}(X)\{l\} \simeq\left(\mathbb{Q}_{l} / \mathbb{Z}_{l}\right)^{2}
$$

pour $l \equiv-1(\bmod 3)$ [Schoen 1995, Theorem 0.1]. Le groupe $\operatorname{Griff}^{2}(X)$ est défini comme le quotient du groupe des cycles à coefficients entiers qui sont homologiquement équivalents à zéro par le sous-groupe de ceux qui sont algébriquement équivalents à zéro. Commençons par clarifier le lien entre ce groupe et $\operatorname{Griff}^{2}\left(X, \mathbb{Z}_{l}\right)$ : 
Proposition 5.4. Soient $k_{0}$ un corps fini de clôture algébrique $k, X_{0} \in B_{\text {Tate }}\left(k_{0}\right)$ et $X=X_{0} \otimes_{k_{0}} k$. Alors, pour tout $n \geq 0$, l'homomorphisme évident

$$
\operatorname{Griff}^{n}(X) \otimes \mathbb{Z}_{l} \rightarrow \operatorname{Griff}^{n}\left(X, \mathbb{Z}_{l}\right)
$$

est bijectif.

Démonstration. Soit $A$ un groupe abélien quelconque. Pour une variété lisse $X$ sur un corps quelconque, on peut définir les cycles à coefficients dans $A$ modulo l'équivalence rationnelle, ou algébrique. Notons ces groupes $C H^{*}(X, A)$ et $A_{\mathrm{alg}}^{*}(X, A)$. Je dis que les homomorphismes

$$
\begin{gathered}
C H^{*}(X) \otimes A \rightarrow C H^{*}(X, A), \\
A_{\text {alg }}^{*}(X) \otimes A \rightarrow A_{\text {alg }}^{*}(X, A)
\end{gathered}
$$

sont bijectifs : par exemple on peut décrire $A_{\text {alg }}^{n}(X, A)$ comme le conoyau d'un homomorphisme

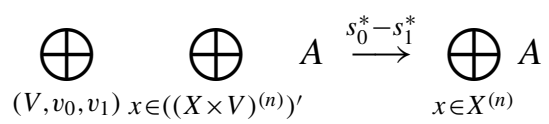

où $\left(V, v_{0}, v_{1}\right)$ décrit l'ensemble des classes d'isomorphismes de $k$-variétés lisses $V$ munies de deux points rationnels $v_{0}$ et $v_{1}$.

Plaçons-nous maintenant dans la situation de la proposition. Notons $C H^{n}(X)_{\text {hom }}$ le noyau de $\mathrm{cl}^{n}: C H^{n}(X) \rightarrow H_{\text {cont }}^{2 n}\left(X, \mathbb{Z}_{l}(n)\right)$. Je dis que l'isomorphisme

$$
C H^{n}(X) \otimes \mathbb{Z}_{l} \stackrel{\sim}{\longrightarrow} C H^{n}\left(X, \mathbb{Z}_{l}\right)
$$

envoie $C H^{n}(X)_{\text {hom }} \otimes \mathbb{Z}_{l}$ sur $C H^{n}\left(X, \mathbb{Z}_{l}\right)_{\text {hom. }}$. En effet, soit $x \in C H^{n}\left(X, \mathbb{Z}_{l}\right)_{\text {hom. }}$ Écrivant $x=\sum \alpha_{i} x_{i}$ avec $\alpha_{i} \in \mathbb{Z}_{l}, x_{i} \in C H^{n}(X)$, on peut (quitte à augmenter $k_{0}$ ) supposer que $x$ provient de $x_{0} \in C H^{n}\left(X_{0}, \mathbb{Z}_{l}\right)$. On a évidemment $x_{0} \in C H^{n}\left(X_{0}, \mathbb{Z}_{l}\right)_{\text {hom }} ;$ le même raisonnement que dans la preuve du théorème 5.2 (utilisant le fait que $\left.X_{0} \in B_{\text {Tate }}\left(k_{0}\right)\right)$ montre alors que $x_{0}$ est de torsion. Mais, pour tout groupe abélien $M$, on a des isomorphismes

$$
M\{l\} \stackrel{\sim}{\longrightarrow} M\{l\} \otimes \mathbb{Z}_{l} \stackrel{\sim}{\longrightarrow}\left(M \otimes \mathbb{Z}_{l}\right)\{l\}
$$

puisque $(M / M\{l\}) \otimes \mathbb{Z}_{l}$ est sans $l$-torsion. Donc $x_{0} \in C H^{n}\left(X_{0}\right)_{\text {hom }}$ et $x \in C H^{n}(X)_{\text {hom }}$. Il résulte de ceci que l'homomorphisme induit

$$
\operatorname{Griff}^{n}(X) \otimes \mathbb{Z}_{l}=A_{\text {alg }}^{n}(X)_{\text {hom }} \otimes \mathbb{Z}_{l} \rightarrow A_{\text {alg }}^{n}\left(X, \mathbb{Z}_{l}\right)_{\text {hom }}=\operatorname{Griff}^{n}\left(X, \mathbb{Z}_{l}\right)
$$

est surjectif, donc bijectif, d'où l'énoncé.

Proposition 5.5. Sous les hypothèses de la proposition 5.4, les conditions suivantes sont équivalentes :

(i) $H^{0}\left(X, \mathscr{H}^{3}\left(\mathbb{Q}_{l} / \mathbb{Z}_{l}(2)\right)\right)$ est fini. 
(ii) Le monomorphisme $\operatorname{Griff}^{2}\left(X, \mathbb{Z}_{l}\right) \rightarrow H_{\mathrm{tr}}^{3}\left(X, \mathbb{Z}_{l}(2)\right) \otimes \mathbb{Q}_{l} / \mathbb{Z}_{l} d u$ théorème $\left.5.2 b\right)$ est surjectif.

(iii) corang $\operatorname{Griff}^{2}\left(X, \mathbb{Z}_{l}\right) \geq \operatorname{dim} H_{\mathrm{tr}}^{3}\left(X, \mathbb{Q}_{l}(2)\right)$.

(iv) L'application b de (4-2) est nulle.

(v) $H_{\mathrm{tr}}^{3}\left(X, \mathbb{Q}_{l} / \mathbb{Z}_{l}(2)\right)$ est fini, quotient de $H_{\mathrm{cont}}^{4}\left(X, \mathbb{Z}_{l}\right)_{\text {tors. }}$.

Si $X$ est une variété abélienne (il suffit que $H^{3}\left(X, \mathbb{Z}_{l}\right)$ et $H^{4}\left(X, \mathbb{Z}_{l}\right)$ soient sans torsion), ces conditions sont encore équivalentes à :

(vi) Pour tout $n \geq 1$, l'homomorphisme $H_{\mathrm{ett}}^{3}\left(X, \mathbb{Z} / l^{n}\right) \rightarrow H_{\mathrm{ett}}^{3}\left(k(X), \mathbb{Z} / l^{n}\right)$ est nul.

Démonstration. Les équivalences (i) $\Longleftrightarrow$ (ii) $\Longleftrightarrow$ (iii) résultent du théorème 5.2 b) et du théorème 1.1. Les équivalences (ii) $\Longleftrightarrow$ (iv) $\Longleftrightarrow$ (v) résultent du théorème $5.2 \mathrm{a}$ ), du corollaire 4.15 et de la proposition 4.14 (ou plus directement du diagramme $(4-2))$.

Si (vi) est vrai, il est vrai stablement (c'est-à-dire à coefficients $\mathbb{Q}_{l} / \mathbb{Z}_{l}$ ), ce qui est équivalent à la nullité de $H_{\mathrm{tr}}^{3}\left(X, \mathbb{Q}_{l} / \mathbb{Z}_{l}\right)$, d'où (iv). Réciproquement, montrons que $(\mathrm{v}) \Longrightarrow(\mathrm{vi})$ si $H_{\text {cont }}^{3}\left(X, \mathbb{Z}_{l}\right)$ et $H_{\text {cont }}^{4}\left(X, \mathbb{Z}_{l}\right)$ sont sans torsion. De (v) on déduit que $H_{\mathrm{tr}}^{3}\left(X, \mathbb{Q}_{l} / \mathbb{Z}_{l}(2)\right)=0$, ce qui donne (vi) stablement. Pour l'obtenir à coefficients finis, considérons le diagramme commutatif aux lignes exactes :

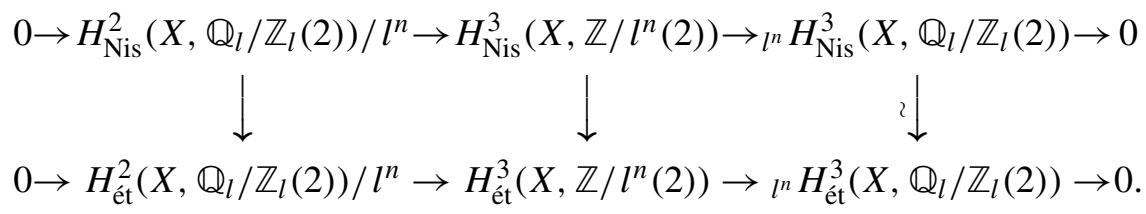

Comme $H_{\text {cont }}^{3}\left(X, \mathbb{Z}_{l}\right)$ est sans torsion, $H_{\mathrm{ett}}^{2}\left(X, \mathbb{Q}_{l} / \mathbb{Z}_{l}(2)\right)$ est divisible et le terme en bas à gauche est nul. La flèche verticale centrale est donc surjective, ce qui donne l'énoncé pour $H_{\text {êt }}^{3}\left(X, \mathbb{Z} / l^{n}(2)\right)$.

Théorème 5.6. Soient $p$ un nombre premier $\equiv 1(\bmod 3), k=\overline{\mathbb{F}}_{p}$, et $E$ la courbe elliptique sur $k$ d'équation $x^{3}+y^{3}+z^{3}=0$. Posons $X=E^{3}$. Si $l \equiv-1(\bmod 3)$, les conditions de la proposition 5.5 sont vérifiées.

Démonstration. Pour commencer, observons que $X \in B_{\text {Tate }}\left(\mathbb{F}_{p}\right)$. Cela résulte du théorème de Spieß [1999], ou simplement de [Soulé 1984, théorème 3] puisque $\operatorname{dim} X=3$.

Montrons (iii). D'après [Schoen 1995, Theorem 0.1] et la proposition 5.4, on a $\operatorname{Griff}^{2}\left(X, \mathbb{Z}_{l}\right) \simeq\left(\mathbb{Q}_{l} / \mathbb{Z}_{l}\right)^{2} ;$ il faut donc montrer que $H_{\text {tr }}^{3}\left(X, \mathbb{Z}_{l}\right)$ est de rang $\leq 2$. Comme $X$ est une variété abélienne, on a un isomorphisme

$$
\Lambda^{3} H_{\text {cont }}^{1}\left(X, \mathbb{Q}_{l}\right) \stackrel{\sim}{\longrightarrow} H_{\text {cont }}^{3}\left(X, \mathbb{Q}_{l}\right) .
$$

L'hypothèse sur $p$ assure que $E$ est ordinaire (cf. [Schoen 1995, p. 46]). Soient $\alpha, \beta$ les nombres de Weil de $E$ sur $\mathbb{F}_{p}$ : on a $\alpha \beta=p$, et $K:=\mathbb{Q}(\alpha)=\mathbb{Q}\left(\mu_{3}\right)$ (ibid.). 
L'espace vectoriel $H_{\text {cont }}^{1}\left(X, \mathbb{Q}_{l}\right)$ est somme de trois exemplaires de $H_{\text {cont }}^{1}\left(E, \mathbb{Q}_{l}\right)$ : il est donc de rang 6. Soit $\left(v_{1}, v_{2}, v_{3}, w_{1}, w_{2}, w_{3}\right)$ une base du $\mathbb{Q}_{l} \otimes K$-module libre $H_{\text {cont }}^{1}\left(X, \mathbb{Q}_{l}\right) \otimes K$ formée de vecteurs propres pour l'action du Frobenius $\phi$, avec $\phi v_{i}=\alpha v_{i}, \phi w_{i}=\beta w_{i}$. Le $\mathbb{Q}_{l} \otimes K$-module $H_{\text {cont }}^{3}\left(X, \mathbb{Q}_{l}\right) \otimes K$ est libre de rang 20, de base les tenseurs purs de degré 3 construits sur les $v_{i}, w_{j}$. Par construction, cette base $B$ est formée de vecteurs propres pour l'action de Frobenius.

Soit $b \in B$. Si $b \notin\left\{v_{1} \wedge v_{2} \wedge v_{3}, w_{1} \wedge w_{2} \wedge w_{3}\right\}, b$ est divisible par $c=v_{i} \wedge w_{j}$ pour un couple $(i, j)$. La valeur propre de $c \in H_{\text {cont }}^{2}\left(X, \mathbb{Q}_{l}\right) \otimes K$ est égale à $p$; en particulier, $c \in H_{\text {cont }}^{2}\left(X, \mathbb{Q}_{l}\right)$. Par le théorème de Tate (dû dans ce cas particulier à Deuring), $c \otimes \mathbb{Q}_{l}(1) \in H_{\text {cont }}^{2}\left(X, \mathbb{Q}_{l}(1)\right)$ est de la forme $\operatorname{cl}(\gamma)$ pour un diviseur $\gamma \in \operatorname{Pic}(X) \otimes \mathbb{Z}_{l}:$ il en résulte que $b \in N H_{\text {cont }}^{3}\left(X, \mathbb{Q}_{l}\right)$.

Ceci montre que $H_{\mathrm{tr}}^{3}\left(X, \mathbb{Q}_{l}\right) \otimes K$ est engendré par $b=v_{1} \wedge v_{2} \wedge v_{3}$ et $b^{\prime}=$ $w_{1} \wedge w_{2} \wedge w_{3}$, et donc que $\operatorname{dim} H_{\text {tr }}^{3}\left(X, \mathbb{Q}_{l}\right) \leq 2$.

Remarque 5.7. Comme $H_{\text {cont }}^{*}\left(X, \mathbb{Z}_{l}\right) \rightarrow H_{\mathrm{et}}^{*}\left(X, \mathbb{Z} / l^{n}\right)$ est surjectif, le calcul fait dans la preuve du théorème 5.6 montre a priori que l'image de $H_{\text {ét }}^{3}\left(X, \mathbb{Z} / l^{n}\right)$ dans $H^{3}\left(F, \mathbb{Z} / l^{n}\right)$ est de rang $\leq 2$, où $F=k(X)$. On aimerait bien démontrer sa nullité (l'énoncé (vi) de la proposition 5.5) directement : il s'agit de voir que, si $x_{1}, x_{2}, x_{2} \in H_{\text {êt }}^{1}\left(X, \mathbb{Z} / l^{n}\right)$, le cup-produit $x_{1} \cdot x_{2} \cdot x_{3}$ est nul dans $H_{\text {êt }}^{3}\left(F, \mathbb{Z} / l^{n}\right)$.

On peut se limiter aux triplets $\left(x_{1}, x_{2}, x_{3}\right)$ tels que

$$
x_{i} \in \operatorname{Im}\left(H_{\text {êt }}^{1}\left(E, \mathbb{Z} / l^{n}\right) \stackrel{\pi_{i}^{*}}{\longrightarrow} H_{\text {êt }}^{1}\left(X, \mathbb{Z} / l^{n}\right)\right)
$$

pour une valeur de $i$, où $\pi_{i}$ est la $i$-ème projection, et sans perte de généralité, supposer $i=1$. Alors $x_{1}$ définit une isogénie $f: E^{\prime} \rightarrow E$ de degré $l^{n}$. Soit $F^{\prime}=k\left(X^{\prime}\right)$, où $X^{\prime}=E^{\prime} \times E \times E$ : d'après Merkurjev-Suslin, la nullité de $x_{1} \cdot x_{2} \cdot x_{3}$ dans $H_{\text {ét }}^{3}\left(F, \mathbb{Z} / l^{n}\right)$ équivaut au fait que $x_{2} \cdot x_{3} \in H^{2}\left(F, \mathbb{Z} / l^{n}\right) \simeq l^{n} B r(F)$ est une norme dans l'extension $F^{\prime} / F$. Peut-on montrer ceci directement?

5D. Autres corps. Si $c d(k) \leq 1$, la suite exacte (5-2) persiste [Jannsen 1988, Theorem 3.3]. Malheureusement, elle ne semble pas apporter d'informations supplémentaires très utiles, sauf peut-être dans le cas d'un corps quasi-fini que je n'ai pas exploré.

Considérons les notations de la preuve de la proposition 3.6. Si $k$ est de type fini mais n'est pas fini, je ne sais pas s'il faut espérer que $K$ est de torsion, même sous toutes les conjectures habituelles (Jannsen le suggère dans [Jannsen 1994, Lemma 2.7]). On peut remplacer $H_{\text {cont }}^{4}\left(X, \mathbb{Z}_{l}(2)\right)$ par le groupe plus fin

$$
\tilde{H}_{\text {cont }}^{4}\left(X, \mathbb{Z}_{l}(2)\right)=\lim _{\longrightarrow} H_{\text {cont }}^{4}\left(\mathscr{X}, \mathbb{Z}_{l}(2)\right)
$$

où $\mathscr{X}$ décrit les modèles réguliers de $X$, de type fini sur Spec $\mathbb{Z}$ (cf. [Jannsen 1990, (11.6.1)]). En caractéristique $p$, par passage à la limite, la conjecture de TateBeilinson implique alors que $K_{\text {ét }}$ est de torsion [Kahn 2005, théorème 60, (iii)]. De 
plus, cette conjecture implique que $C H^{2}(X)$ est de type fini (comme quotient de $C H^{2}(\mathscr{C})$ pour un modèle $\mathscr{X}$ lisse de type fini), donc que $K$ est fini. Par contre, elle n'implique pas a priori que $H_{\mathrm{ett}}^{4}(X, \mathbb{Z}(2))$ est de type fini (dans les suites exactes de Gysin pour un diviseur, le terme suivant est de la forme $\left.H_{\text {ét }}^{3}(Z, \mathbb{Z}(1))=B r(Z) \ldots\right)$ donc il se pourrait fort bien que $K_{\text {ét }}$ ait une partie divisible non triviale.

Le bon objet avec lequel travailler pour des variétés ouvertes sur un corps fini est $H_{W}^{4}(\mathscr{X}, \mathbb{Z}(2))$ (cohomologie Weil-étale) : c'est celui qui permet d'attraper tout $\tilde{H}_{\text {cont }}^{4}\left(X, \mathbb{Z}_{l}(2)\right)$ pour $l \neq p$ [Kahn 2005, théorème 64]. Mais cela a l'air compliqué, cf. [Kahn 2003, (3.2)] ou [Kahn 2005, théorème 62 (ii)].

\section{Annexe : Cohomologie de Hodge-Witt logarithmique sur des corps imparfaits}

Dans [Geisser et Levine 2000], Geisser et Levine comparent la cohomologie motivique modulo $p$ d'un corps de caractéristique $p$ quelconque avec sa cohomologie de Hodge(-Witt) logarithmique, mais n'en déduisent une comparaison globale que pour des variétés lisses sur un corps parfait. Le but de ce numéro est de rappeler les bases de cette comparaison et de se débarrasser de manière «triviale » de l'hypothèse de perfection, à l'aide d'une observation classique de Quillen [1973, p. 133, démonstration du théorème 5.11].

Cohomologie de Hodge-Witt logarithmique. Soit $X$ un schéma de caractéristique p. On lui associe son pro-complexe de de Rham-Witt [Illusie 1979, p. 548, 1.12]

$$
\left(W_{r} \Omega_{X}^{*}\right)_{r \geq 1}
$$

qui est un système projectif de faisceaux d'algèbres différentielles graduées sur $X_{\text {ét }}$, prolongeant (pour $\cdot=0$ ) le pro-faisceau des vecteurs de Witt et (pour $r=1$ ) le complexe des différentielles de Kähler. Il est muni d'un opérateur $F: W_{r} \Omega_{X}^{n} \rightarrow$ $W_{r-1} \Omega_{X}^{n}$ [Illusie 1979, p. 562, théorème 2.17].

Si $X$ est défini sur un corps parfait $k$, on a évidemment

$$
W_{r} \Omega_{X}^{*}=W_{r} \Omega_{X / k}^{*}
$$

On a des applications « de Teichmüller» (multiplicatives)

$$
\mathfrak{O}_{X} \rightarrow W_{r} \mathscr{O}_{X}, \quad x \mapsto \underline{x}=(x, 0, \ldots, 0, \ldots)
$$

[Illusie 1979, p. 505, (1.1.7)], qu'on utilise pour définir les homomorphismes

$$
d \log : \mathbb{O}_{X}^{*} /\left(\mathbb{O}_{X}^{*}\right)^{p^{r}} \rightarrow W_{r} \Omega_{X}^{1}, \quad x \mapsto d \underline{x} / \underline{x}
$$

[Illusie 1979, p. 580, (3.23.1)]. On définit alors $W_{r} \Omega_{X, \log }^{n}$ comme le sous-faisceau de $W_{r} \Omega_{X}^{n}$ engendré localement pour la topologie étale par les sections de la forme 
$d \log x_{1} \wedge \cdots \wedge d \log x_{n}$ [Illusie 1979, p. 596, (5.7.1)]; comme dans [Geisser et Levine 2000], nous noterons simplement ce faisceau $v_{r}(n)_{X}$.

Lemme A.1. Pour tout $x \in \Gamma\left(X, O_{X}\right)$ et pour tout $r \geq 1$, on a

$$
\underline{x} \wedge \underline{1-x}=0 \in \Gamma\left(X, W_{r} \Omega_{X}^{2}\right) .
$$

Démonstration. (Illusie) Le morphisme $X \rightarrow \mathbb{A}_{\mathbb{F}_{p}}^{1}$ défini par $x$ nous ramène au cas universel $X=\operatorname{Spec} \mathbb{F}_{p}[t], x=t$. Mais alors $W_{r} \Omega_{X}^{2}=0$ puisque $\operatorname{dim} X=1$.

Le symbole logarithmique. Supposons $X=\operatorname{Spec} k$, où $k$ est un corps. Le lemme A.1 implique que l'homomorphisme $d \log$ de (A-1) induit un symbole logarithmique

$$
\begin{aligned}
d \log : K_{n}^{M}(k) / p^{r} & \rightarrow v_{r}(n)_{k}, \\
\left\{x_{1}, \ldots, x_{n}\right\} & \mapsto d \log \left(x_{1}\right) \wedge \cdots \wedge d \log \left(x_{n}\right) .
\end{aligned}
$$

Soit $K$ le corps des fonctions d'un $k$-schéma lisse $X$, où $k$ est parfait de caractéristique $p$. Un point $x$ de codimension 1 de $X$ définit une valuation discrète $v$ sur $K$, de corps résiduel $E=k(x)$. Le théorème de pureté de Gros [1985, p. 46, théorème 3.5.8] et la longue suite exacte de cohomologie à supports définissent des homomorphismes résidus

$$
v_{r}(n)_{K} \stackrel{\partial_{v}}{\longrightarrow} v_{r}(n-1)_{E} .
$$

Lemme A.2. Le diagramme

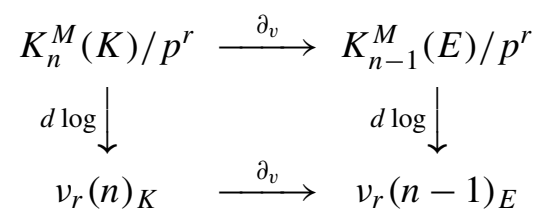

où la flèche horizontale du haut est le résidu en $K$-théorie de Milnor, est commutatif au signe près.

Démonstration. Pour $n=1,2$ c'est fait dans [Gros et Suwa 1988b, p. 625, lemme 4.11]. La démonstration ne se propage pas tout à fait à $n>2$ car elle utilise la formule explicite donnant $\partial(\{x, y\})$ pour $x, y \in K^{*}$. Pour la propager, il suffit toutefois de remarquer que $K_{n}^{M}(K)$ est engendré par les symboles de la forme $\left\{u_{1}, \ldots, u_{n-1}, x\right\}$ avec $u_{i} \in O_{v}^{*}$ et $x \in K^{*}$.

Le morphisme de comparaison. Supposons $X$ lisse sur un corps parfait $k$. D'après [Illusie 1979, p. 597, théorème 5.7.2], on a une suite exacte de pro-faisceaux étales

$$
0 \rightarrow v \cdot(n)_{X} \rightarrow W \cdot \Omega_{X}^{n} \stackrel{1-F}{\longrightarrow} W \cdot \Omega_{X}^{n} \rightarrow 0
$$

qui en fait n'interviendra pas ici. De plus, on a le théorème suivant, dû à Gros et Suwa : 
Théorème A.3. On a une suite exacte de faisceaux zariskiens

$$
0 \rightarrow \alpha_{*} v_{r}(n)_{X} \rightarrow \bigoplus_{x \in X^{(0)}}\left(v_{r}(n)_{k(x)}\right)_{\overline{\{x\}}} \stackrel{\partial}{\longrightarrow} \bigoplus_{x \in X^{(1)}}\left(v_{r}(n-1)_{k(x)}\right)_{\{x\}} \stackrel{\partial}{\longrightarrow} \cdots
$$

où $\alpha$ désigne la projection $X_{\text {ét }} \rightarrow X_{\mathrm{Zar}}$ et les différentielles $\partial$ sont construites à partir des résidus (A-3).

Démonstration. Voir [Gros et Suwa 1988b, corollaire 1.6] ou [Colliot-Thélène et al. 1997, p. 70, Example 7.4 (3)].

Supposons maintenant $X$ régulier de type fini sur un corps $k$ (de caractéristique $p$ ). Supposons d'abord $k$ de type fini sur $\mathbb{F}_{p}:$ alors $X$ admet un modèle $\mathscr{X}$ régulier, donc lisse, de type fini sur $\mathbb{F}_{p}$. Soit $j: X \rightarrow \mathscr{X}$ la pro-immersion ouverte correspondante : on a évidemment

$$
v_{r}(n)_{X}=j^{*} v_{r}(n)_{\mathscr{X}}
$$

puisque les anneaux semi-locaux de $X$ sont certains anneaux semi-locaux de $\mathscr{X}$.

Interprétons maintenant $K_{n}^{M}(K) / p^{n}$ comme $H^{n}(K, \mathbb{Z} / p(n))$, cf. théorème 2.3. $\mathrm{Vu}$ la remarque 2.4 et le théorème A.3, les homomorphismes (A-2) et le lemme A.2 induisent des homomorphismes de faisceaux

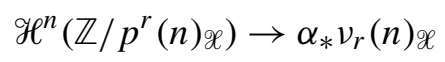

et donc des morphismes dans $D^{-}\left(\mathscr{X}_{\mathrm{Zar}}\right)$

$$
\mathbb{Z} / p^{r}(n)_{\mathscr{X}} \rightarrow \alpha_{*} v_{r}(n) \mathscr{x}[-n]
$$

puisque $\mathscr{H}^{i}\left(\mathbb{Z} / p^{r}(n)_{\mathscr{X}}\right)=0$ pour $i>n$ (lemme 2.5$)$.

D'où, en appliquant $j^{*}$, des morphismes dans $D^{-}\left(X_{\mathrm{Zar}}\right)$

$$
\mathbb{Z} / p^{r}(n)_{X} \rightarrow \alpha_{*} v_{r}(n)_{X}[-n] .
$$

Si $k$ est quelconque, écrivons

$$
k=\underset{\alpha}{\lim _{\alpha}} k_{\alpha} \quad \text { et } \quad X={\underset{\lim }{\alpha}}_{\alpha} X_{\alpha}
$$

où les $k_{\alpha}$ sont de type fini sur $\mathbb{F}_{p}$ et $X_{\alpha}$ est un $k_{\alpha}$-schéma régulier de type fini, de sorte que $k_{\alpha} \subset k_{\beta}$ induise un isomorphisme $X_{\beta} \stackrel{\sim}{\longrightarrow} X_{\alpha} \otimes_{k_{\alpha}} k_{\beta}$. On a évidemment :

$$
\begin{aligned}
v_{r}(n)_{X} & =\underset{\alpha}{\lim } \pi_{\alpha}^{*} v_{r}(n)_{X_{\alpha}}, \\
\mathcal{H}^{n}\left(\mathbb{Z} / p^{r}(n)_{X}\right) & =\underset{\alpha}{\lim } \pi_{\alpha}^{*} \mathcal{H}^{n}\left(\mathbb{Z} / p^{r}(n)_{X_{\alpha}}\right),
\end{aligned}
$$

où $\pi_{\alpha}: X \rightarrow X_{\alpha}$ est le morphisme canonique. Ceci étend la définition de (A-4) au cas où le corps de base est quelconque. On voit de même : 
Proposition A.4 (cf. [Quillen 1973, démonstration du théorème 5.11]). La suite exacte du théorème A.3 s'étend à tout $X$ régulier de type fini sur un corps.

\section{Le théorème de Geisser-Levine.}

Théorème A.5. Soit $X$ un schéma régulier de type fini sur un corps $k$ de caractéristique p. Alors le morphisme (A-4) est un isomorphisme.

Démonstration. Il s'agit de voir que

$$
\mathscr{H}^{i}\left(\mathbb{Z} / p^{r}(n)_{X}\right) \simeq \begin{cases}0 & \text { si } i \neq n, \\ \alpha_{*} v_{r}(n)_{X} & \text { si } i=n,\end{cases}
$$

le dernier isomorphisme étant induit par (A-4). L'énoncé est clair pour $i>n$, cf. lemme 2.5.

1) $X=$ Spec $k$ : c'est le théorème de Bloch-Gabber-Kato pour $i=n$ [Bloch et Kato 1986, p. 117, corollaire 2.8] et celui de Geisser-Levine [2000, théorème 1.1] pour $i<n$.

2) $X$ lisse sur $k$ parfait : on se réduit à 1 ) en utilisant le théorème A.3, le lemme A.2 et la conjecture de Gersten pour la cohomologie motivique, cf. preuve du lemme 2.5. 3) $k$ de type fini sur $\mathbb{F}_{p}$ : on se ramène à 2) par la technique du numéro précédent. 4) $k$ quelconque : on se ramène à 3) par passage à la limite.

Remarque A.6. On pourrait court-circuiter les étapes 2) et 3), dans l'esprit de la proposition A.4.

Corollaire A.7. Soit $X$ un schéma régulier de type fini sur un corps $k$ de caractéristique $p$. Alors le morphisme $\alpha^{*}(\mathrm{~A}-4)$ est un isomorphisme, où $\alpha$ est la projection $X_{\text {ét }} \rightarrow X_{\text {Zar }}$.

\section{Remerciements}

Cet article est l'élaboration d'un texte rédigé en janvier 2010, lui-même directement inspiré de plusieurs discussions avec Jean-Louis Colliot-Thélène autour de son travail avec Claire Voisin [Colliot-Thélène et Voisin 2010], alors en cours d'achèvement. Il a ensuite bénéficié de la lecture de [Colliot-Thélène et Voisin 2010], ainsi que de discussions avec Colliot-Thélène autour d'un article commun en projet [Colliot-Thélène et Kahn 2011] : je le remercie de sa lecture critique de versions préliminaires de ce texte, et notamment de m'avoir fait remarquer qu'il n'est pas nécessaire de supposer $k$ de type fini sur son sous-corps premier dans le théorème 1.1. Je remercie l'IMPA de Rio de Janeiro pour son hospitalité pendant la fin de sa rédaction, ainsi que la coopération franco-brésilienne pour son soutien. Pour finir, je remercie Luc Illusie de m'avoir indiqué une démonstration (triviale !) du lemme A.1, lemme qui à ma connaissance ne figure nulle part dans la littérature. 


\section{Bibliographie}

[Bloch 1986] S. Bloch, "Algebraic cycles and higher K-theory", Adv. in Math. 61:3 (1986), 267-304. MR 88f:18010 Zbl 0608.14004

[Bloch 1994] S. Bloch, "The moving lemma for higher Chow groups", J. Algebraic Geom. 3:3 (1994), 537-568. MR 96c:14007 Zbl 0830.14003

[Bloch 2010] S. Bloch, Lectures on algebraic cycles, 2nd éd., New Mathematical Monographs 16, Cambridge University, 2010. MR 2011h:14009 Zbl 1201.14006

[Bloch et Kato 1986] S. Bloch et K. Kato, "p-adic étale cohomology”, Inst. Hautes Études Sci. Publ. Math. 63 (1986), 107-152. MR 87k:14018 Zbl 0613.14017

[Bloch et Ogus 1974] S. Bloch et A. Ogus, "Gersten's conjecture and the homology of schemes", Ann. Sci. École Norm. Sup. (4) 7 (1974), 181-201. MR 54 \#318 Zbl 0307.14008

[Bloch et Srinivas 1983] S. Bloch et V. Srinivas, "Remarks on correspondences and algebraic cycles", Amer. J. Math. 105:5 (1983), 1235-1253. MR 85i:14002 Zbl 0525.14003

[Block 1995] S. Block, “A spectral sequence for motivic cohomology”, preprint, 1995, Voir http :// www.math.uiuc.edu/K-theory/0062/.

[Colliot-Thélène et Kahn 2011] J. Colliot-Thélène et B. Kahn, "Cycles de codimension 2 et $H^{3}$ non ramifié pour les variétés sur les corps finis", preprint, 2011. arXiv 1104.3350v1

[Colliot-Thélène et Raskind 1985] J.-L. Colliot-Thélène et W. Raskind, " $\mathscr{K}_{2}$-cohomology and the second Chow group”, Math. Ann. 270:2 (1985), 165-199. MR 86m:14005 Zbl 0536.14004

[Colliot-Thélène et Voisin 2010] J. Colliot-Thélène et C. Voisin, "Cohomologie non ramifiée et conjecture de Hodge entière", preprint, 2010. arXiv 1005.2778v1

[Colliot-Thélène et al. 1983] J.-L. Colliot-Thélène, J.-J. Sansuc et C. Soulé, “Torsion dans le groupe de Chow de codimension deux", Duke Math. J. 50:3 (1983), 763-801. MR 85d:14010 Zbl 0574.14004

[Colliot-Thélène et al. 1997] J.-L. Colliot-Thélène, R. T. Hoobler et B. Kahn, "The Bloch-OgusGabber theorem”, pp. 31-94 dans Algebraic K-theory (Toronto, 1996), édité par V. P. Snaith, Fields Inst. Commun. 16, Amer. Math. Soc., Providence, RI, 1997. MR 98j:14021 Zbl 0911.14004

[Deligne 1974] P. Deligne, "La conjecture de Weil, I", Inst. Hautes Études Sci. Publ. Math. 43 (1974), 273-307. MR 49 \#5013 Zbl 0287.14001

[Deninger 1988] C. Deninger, "A proper base change theorem for nontorsion sheaves in étale cohomology”, J. Pure Appl. Algebra 50:3 (1988), 231-235. MR 89f:14016 Zbl 0672.14010

[Geisser et Levine 2000] T. Geisser et M. Levine, "The $K$-theory of fields in characteristic $p$ ", Invent. Math. 139:3 (2000), 459-493. MR 2001f:19002 Zbl 0957.19003

[Geisser et Levine 2001] T. Geisser et M. Levine, "The Bloch-Kato conjecture and a theorem of Suslin-Voevodsky”, J. Reine Angew. Math. 530 (2001), 55-103. MR 2003a:14031 Zbl 1023.14003

[Gros 1985] M. Gros, Classes de Chern et classes de cycles en cohomologie de Hodge-Witt logarithmique, Mém. Soc. Math. France (N.S.) 21, 1985. MR 87m:14021 Zbl 0615.14011

[Gros et Suwa 1988a] M. Gros et N. Suwa, "Application d'Abel-Jacobi p-adique et cycles algébriques”, Duke Math. J. 57:2 (1988), 579-613. MR 89h:14006a Zbl 0697.14005

[Gros et Suwa 1988b] M. Gros et N. Suwa, "La conjecture de Gersten pour les faisceaux de HodgeWitt logarithmique”, Duke Math. J. 57:2 (1988), 615-628. MR 89h:14006b Zbl 0715.14011 
[Grothendieck 1968] A. Grothendieck, "Le groupe de Brauer, III : Exemples et compléments", pp. 88-188 dans Dix exposés sur la cohomologie des schémas, Advanced Studies Pure Math. 3, North-Holland, Amsterdam, 1968. MR 39 \#5586c Zbl 0198.25901

[Illusie 1979] L. Illusie, "Complexe de de Rham-Witt et cohomologie cristalline", Ann. Sci. École Norm. Sup. (4) 12:4 (1979), 501-661. MR 82d:14013

[Jannsen 1988] U. Jannsen, “Continuous étale cohomology”, Math. Ann. 280:2 (1988), 207-245. MR 89a:14022 Zbl 0649.14011

[Jannsen 1990] U. Jannsen, Mixed motives and algebraic $K$-theory, Lecture Notes in Mathematics 1400, Springer, Berlin, 1990. MR 91g:14008 Zbl 0691.14001

[Jannsen 1992] U. Jannsen, "Motives, numerical equivalence, and semi-simplicity", Invent. Math. 107:3 (1992), 447-452. MR 93g:14009 Zbl 0762.14003

[Jannsen 1994] U. Jannsen, "Motivic sheaves and filtrations on Chow groups", pp. 245-302 dans Motives (Seattle, WA, 1991), édité par U. Jannsen et al., Proc. Sympos. Pure Math. 55, Amer. Math. Soc., Providence, RI, 1994. MR 95c:14006 Zbl 0811.14004

[de Jong 1996] A. J. de Jong, "Smoothness, semi-stability and alterations", Inst. Hautes Études Sci. Publ. Math. 83 (1996), 51-93. MR 98e:14011 Zbl 0916.14005

[Kahn 1996] B. Kahn, "Applications of weight-two motivic cohomology”, Doc. Math. 1 (1996), 395-416. MR 98b:14007 Zbl 0883.19002

[Kahn 1997] B. Kahn, "La conjecture de Milnor (d'après V. Voevodsky)", pp. 379-418 dans Séminaire Bourbaki, Astérisque 245, Société Mathématique de France, Paris, 1997. Reprinted as pp. 1105-1149 in Handbook of K-theory, Springer, Berlin, 2005. MR 1627119 Zbl 0916.19001

[Kahn 2002] B. Kahn, "The Geisser-Levine method revisited and algebraic cycles over a finite field", Math. Ann. 324:3 (2002), 581-617. MR 2003h:14034 Zbl 1014.14004

[Kahn 2003] B. Kahn, "Équivalences rationnelle et numérique sur certaines variétés de type Abélien sur un corps fini”, Ann. Sci. École Norm. Sup. (4) 36:6 (2003), 977-1002. MR 2005e:14037 Zbl 1073.14034

[Kahn 2005] B. Kahn, "Algebraic $K$-theory, algebraic cycles and arithmetic geometry”, pp. 351-428 dans Handbook of K-theory, vol. 1, édité par E. M. Friedlander et D. R. Grayson, Springer, Berlin, 2005. MR 2007b:14016 Zbl 1115.19003

[Kahn 2006] B. Kahn, "Sur le groupe des classes d'un schéma arithmétique”, Bull. Soc. Math. France 134:3 (2006), 395-415. MR 2007d:14052 Zbl 1222.14048

[Kahn et al. 2007] B. Kahn, J. P. Murre et C. Pedrini, "On the transcendental part of the motive of a surface", pp. 143-202 dans Algebraic cycles and motives, vol. 2, édité par J. Nagel et C. Peters, London Math. Soc. Lecture Note Ser. 344, Cambridge Univ., 2007. MR 2009c:14010 Zbl 1130.14008

[Katz et Messing 1974] N. M. Katz et W. Messing, "Some consequences of the Riemann hypothesis for varieties over finite fields”, Invent. Math. 23 (1974), 73-77. MR 48 \#11117 Zbl 0275.14011

[Kimura 2005] S.-I. Kimura, "Chow groups are finite dimensional, in some sense", Math. Ann. 331:1 (2005), 173-201. MR 2005j:14007 Zbl 1067.14006

[Kleiman 1968] S. L. Kleiman, "Algebraic cycles and the Weil conjectures", pp. 359-386 dans Dix exposés sur la cohomologie des schémas, Advanced Studies Pure Math. 3, North-Holland, Amsterdam, 1968. MR 45 \#1920 Zbl 0198.25902 
[Lichtenbaum 1990] S. Lichtenbaum, "New results on weight-two motivic cohomology", pp. 3555 dans The Grothendieck Festschrift, vol. 3, Progr. Math. 88, Birkhäuser, Boston, MA, 1990. MR 92m:14030 Zbl 0809.14004

[Nesterenko et Suslin 1989] Y. P. Nesterenko et A. A. Suslin, "Homology of the general linear group over a local ring, and Milnor's K-theory”, Izv. Akad. Nauk SSSR Ser. Mat. 53:1 (1989), 121-146. In Russian ; translated in Math. USSR-Izv. 34 :1 (1990), 121-145. MR 90a:20092 Zbl 0668.18011

[Quillen 1973] D. Quillen, "Higher algebraic K-theory, I", pp. 85-147 dans Algebraic K-theory, I : Higher K-theories (Seattle, WA, 1972), édité par H. Bass, Lecture Notes in Math. 341, Springer, Berlin, 1973. MR 49 \#2895 Zbl 0292.18004

[Schoen 1995] C. Schoen, "On the computation of the cycle class map for nullhomologous cycles over the algebraic closure of a finite field”, Ann. Sci. École Norm. Sup. (4) 28:1 (1995), 1-50. MR 95j:14010 Zbl 0839.14004

[Schoen 2002] C. Schoen, "Complex varieties for which the Chow group mod $n$ is not finite", $J$. Algebraic Geom. 11:1 (2002), 41-100. MR 2002h:14004 Zbl 1059.14010

[Soulé 1984] C. Soulé, “Groupes de Chow et $K$-théorie de variétés sur un corps fini”, Math. Ann. 268:3 (1984), 317-345. MR 86k:14017 Zbl 0573.14001

[Spaltenstein 1988] N. Spaltenstein, "Resolutions of unbounded complexes", Compositio Math. 65:2 (1988), 121-154. MR 89m:18013 Zbl 0636.18006

[Spieß 1999] M. Spieß, "Proof of the Tate conjecture for products of elliptic curves over finite fields", Math. Ann. 314:2 (1999), 285-290. MR 2000d:14012 Zbl 0941.11026

[Suslin 1987] A. A. Suslin, “Torsion in $K_{2}$ of fields”, K-Theory 1:1 (1987), 5-29. MR 89a:11123 Zbl 0635.12015

[Suslin et Voevodsky 2000] A. Suslin et V. Voevodsky, "Bloch-Kato conjecture and motivic cohomology with finite coefficients", pp. 117-189 dans The arithmetic and geometry of algebraic cycles (Banff, 1998), édité par B. B. Gordon et al., NATO Sci. Ser. C Math. Phys. Sci. 548, Kluwer, Dordrecht, 2000. MR 2001g:14031 Zbl 1005.19001

[Totaro 1992] B. Totaro, "Milnor $K$-theory is the simplest part of algebraic $K$-theory", $K$-Theory $\mathbf{6}: 2$ (1992), 177-189. MR 94d:19009 Zbl 0776.19003

[Voevodsky 2003] V. Voevodsky, "Motivic cohomology with Z/2-coefficients", Publ. Math. Inst. Hautes Études Sci. 98 (2003), 59-104. MR 2005b:14038b Zbl 1057.14028

Communicated by Raman Parimala

Received 2011-04-14 Revised 2011-06-12 Accepted 2011-07-17

kahn@math.jussieu.fr Institut de Mathématiques de Jussieu, CNRS, 4 place Jussieu, Case 247, 75252 Cedex 05 Paris, France 


\section{Algebra \& Number Theory}

msp.berkeley.edu/ant

\section{EDITORS}

MANAGING EDITOR

Bjorn Poonen

Massachusetts Institute of Technology

Cambridge, USA

\author{
EDITORIAL BOARD CHAIR \\ David Eisenbud \\ University of California \\ Berkeley, USA
}

\section{BOARD OF EDITORS}

Georgia Benkart

Dave Benson

Richard E. Borcherds

John H. Coates

J-L. Colliot-Thélène

Brian D. Conrad

Hélène Esnault

Hubert Flenner

Edward Frenkel

Andrew Granville

Joseph Gubeladze

Ehud Hrushovski

Craig Huneke

Mikhail Kapranov

Yujiro Kawamata

János Kollár

Yuri Manin

Barry Mazur

Philippe Michel
University of Wisconsin, Madison, USA

University of Aberdeen, Scotland

University of California, Berkeley, USA

University of Cambridge, UK

CNRS, Université Paris-Sud, France

University of Michigan, USA

Freie Universität Berlin, Germany

Ruhr-Universität, Germany

University of California, Berkeley, USA

Université de Montréal, Canada

San Francisco State University, USA

Hebrew University, Israel

University of Virginia, USA

Yale University, USA

University of Tokyo, Japan

Princeton University, USA

Northwestern University, USA

Harvard University, USA

École Polytechnique Fédérale de Lausanne
Susan Montgomery

Shigefumi Mori

Raman Parimala

Jonathan Pila

Victor Reiner

Karl Rubin

Peter Sarnak

Joseph H. Silverman

Michael Singer

Vasudevan Srinivas

J. Toby Stafford

Bernd Sturmfels

Richard Taylor

Ravi Vakil

Michel van den Bergh

Marie-France Vignéras

Kei-Ichi Watanabe

Andrei Zelevinsky

Efim Zelmanov
University of Southern California, USA

RIMS, Kyoto University, Japan

Emory University, USA

University of Oxford, UK

University of Minnesota, USA

University of California, Irvine, USA

Princeton University, USA

Brown University, USA

North Carolina State University, USA

Tata Inst. of Fund. Research, India

University of Michigan, USA

University of California, Berkeley, USA

Harvard University, USA

Stanford University, USA

Hasselt University, Belgium

Université Paris VII, France

Nihon University, Japan

Northeastern University, USA

University of California, San Diego, USA

\section{PRODUCTION}

production@msp.org

Silvio Levy, Scientific Editor

See inside back cover or www.jant.org for submission instructions.

The subscription price for 2012 is US \$175/year for the electronic version, and \$275/year ( $\$ 40$ shipping outside the US) for print and electronic. Subscriptions, requests for back issues from the last three years and changes of subscribers address should be sent to Mathematical Sciences Publishers, Department of Mathematics, University of California, Berkeley, CA 94720-3840, USA.

Algebra \& Number Theory (ISSN 1937-0652) at Mathematical Sciences Publishers, Department of Mathematics, University of California, Berkeley, CA 94720-3840 is published continuously online. Periodical rate postage paid at Berkeley, CA 94704, and additional mailing offices.

ANT peer review and production are managed by EditFLOW ${ }^{\circledR}$ from Mathematical Sciences Publishers.

PUBLISHED BY

mathematical sciences publishers

http://msp.org/

A NON-PROFIT CORPORATION

Typeset in IATEX

Copyright ( 2012 by Mathematical Sciences Publishers 


\section{Algebra \& Number Theory}

\section{Volume $6 \quad$ No. $7 \quad 2012$}

On the rank of the fibers of rational elliptic surfaces

Cecília Salgado

Néron's pairing and relative algebraic equivalence

CÉDRIC PÉPIN

Free subalgebras of quotient rings of Ore extensions

JASON P. BELL and DANIEL ROGALSKI

Classes de cycles motiviques étales

1369

BRUNO KAHN

Higher-order Maass forms

1409

ROELOF BRUGGEMAN and NiKOLAOS DIAMANTIS

Log canonical thresholds, $F$-pure thresholds, and nonstandard extensions

Bhargav Bhatt, Daniel J. Hernández, Lance Edward Miller and Mircea MUSTAȚĂ

The semistable reduction problem for the space of morphisms on $\mathbb{P}^{n}$

ALON LEVY

Grothendieck's trace map for arithmetic surfaces via residues and higher adèles

MATTHEW MORROW

Crystalline extensions and the weight part of Serre's conjecture

Toby GeE, Tong LiU and DAVID SAVITT

Annihilating the cohomology of group schemes 